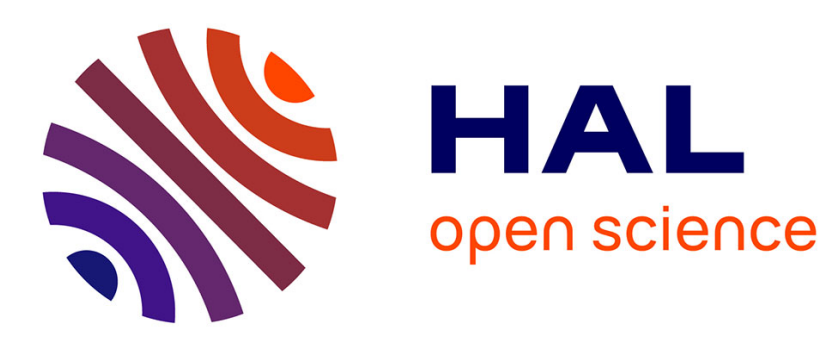

\title{
Design criteria for a printed tissue engineering construct: A mathematical homogenization approach
} R.J. Shipley, G.W. Jones, R.J. Dyson, B.G. Sengers, C.L. Bailey, C.J. Catt, C.P. Please, J. Malda

\section{- To cite this version:}

R.J. Shipley, G.W. Jones, R.J. Dyson, B.G. Sengers, C.L. Bailey, et al.. Design criteria for a printed tissue engineering construct: A mathematical homogenization approach. Journal of Theoretical Biology, 2009, 259 (3), pp.489. 10.1016/j.jtbi.2009.03.037 . hal-00554599

\author{
HAL Id: hal-00554599 \\ https://hal.science/hal-00554599
}

Submitted on 11 Jan 2011

HAL is a multi-disciplinary open access archive for the deposit and dissemination of scientific research documents, whether they are published or not. The documents may come from teaching and research institutions in France or abroad, or from public or private research centers.
L'archive ouverte pluridisciplinaire HAL, est destinée au dépôt et à la diffusion de documents scientifiques de niveau recherche, publiés ou non, émanant des établissements d'enseignement et de recherche français ou étrangers, des laboratoires publics ou privés. 


\section{Author's Accepted Manuscript}

Design criteria for a printed tissue engineering construct: A mathematical homogenization approach

R.J. Shipley, G.W. Jones, R.J. Dyson, B.G. Sengers, C.L. Bailey, C.J. Catt, C.P. Please, J. Malda

PII: $\quad$ S0022-5193(09)00143-X

DOI: $\quad$ doi:10.1016/j.jtbi.2009.03.037

Reference: $\quad$ YJTBI 5519

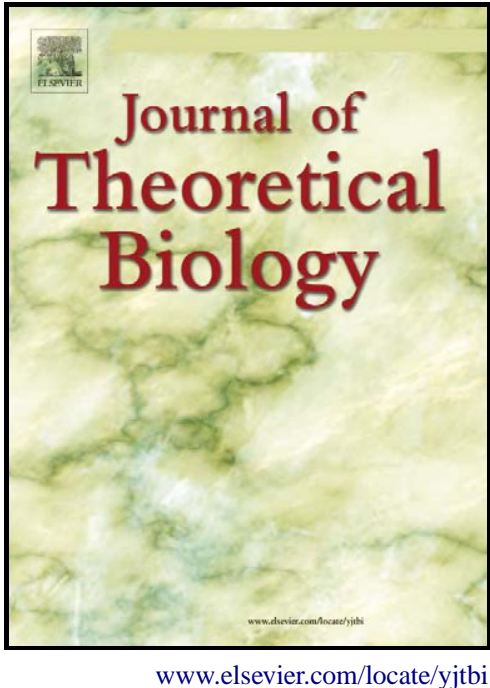

To appear in: $\quad$ Journal of Theoretical Biology

Received date: 27 October 2008

Revised date: $\quad 26$ March 2009

Accepted date: $\quad 28$ March 2009

Cite this article as: R.J. Shipley, G.W. Jones, R.J. Dyson, B.G. Sengers, C.L. Bailey, C.J. Catt, C.P. Please and J. Malda, Design criteria for a printed tissue engineering construct: A mathematical homogenization approach, Journal of Theoretical Biology (2009), doi:10.1016/j.jtbi.2009.03.037

This is a PDF file of an unedited manuscript that has been accepted for publication. As a service to our customers we are providing this early version of the manuscript. The manuscript will undergo copyediting, typesetting, and review of the resulting galley proof before it is published in its final citable form. Please note that during the production process errors may be discovered which could affect the content, and all legal disclaimers that apply to the journal pertain. 
manuscript No.

(will be inserted by the editor)

\title{
Design Criteria For A Printed Tissue Engineering Construct: A Mathematical Homogenization Approach
}

\author{
RJ Shipley · GW Jones · RJ Dyson • BG Sengers • \\ CL Bailey · CJ Catt • CP Please • J Malda
}

Received: date / Accepted: date

Abstract Cartilage tissue repair procedures currently under development aim to create a construct in which patient-derived cells are seeded and expanded ex vivo before implantation back into the body. The key challenge is producing physiologically realistic constructs that mimic real tissue structure and function. One option with vast potential is to print strands of material in a 3D structure called a scaffold that imitates the real tissue structure; the strands are composed of gel seeded with cells and so provide a template for cartilaginous tissue growth. The scaffold is placed in the construct and pumped with nutrient-rich culture medium to supply nutrients to the cells and remove waste products, thus promoting tissue growth.

In this paper we use asymptotic homogenization to determine the effective flow and transport properties of such a printed scaffold system. These properties are used to predict the distribution of nutrient/waste products through the construct, and to specify design criteria for the scaffold that will optimise the growth of functional tissue.

Keywords Cartilage; perfusion; scaffold; construct; mathematical modelling; averaging; species transport

RJ Shipley, GW Jones

Oxford Centre for Industrial and Applied Mathematics, Mathematical Institute, $24-29$ St. Giles', Oxford OX1 $3 \mathrm{LB}$

Tel.: +441865 273525

Fax: +44 1865270515

E-mail: shipley@maths.ox.ac.uk

RJ Dyson

Centre for Plant Integrative Biology and School of Mathematical Sciences, University of Nottingham.

BG Sengers

Bone and Joint Research Group, School of Medicine, University of Southampton

CL Bailey

Faculty of Engineering, Loughborough University

CP Please, CJ Catt

School of Mathematics,University of Southampton

J Malda

Department of Orthopaedics, University Medical Centre Utrecht, Utrecht, the Netherlands 


\section{Introduction}

The treatment of damage to articulating joints, such as the knee or hip, is a challenge for orthopaedic surgeons world-wide. It is well established that the articular cartilage covering the surface of articulating joints has limited regenerative capacity, and that any lesions are a major cause of pain, swelling, and mechanical impairment. In addition, if left untreated, damage to the articular cartilage may lead to osteoarthritis (OA), causing considerable disability by limiting employment, sport participation, and activities of daily living.

Regenerative medicine has the potential to provide a solution to these conditions. It is a newly emerging area that aims to restore tissue function by applying principles of engineering and life sciences to develop biological substitutes. Patient-derived cells can be expanded, and cartilage formation can be initiated on biodegradable 3-dimensional scaffold conduits [52]. However, cartilage has proved difficult to engineer and despite the significant efforts $(\approx 1600$ PubMed articles $)$ over the past $15+$ years, there are no clinical approaches that are capable of consistently restoring long-term function to damaged articular cartilage. Traditionally, cartilage has been regarded as a relatively simple tissue to engineer as it is not vascularised but recent research has demonstrated that the true picture is more complicated. For example, the majority of approaches to date aim to create a relatively simple homogeneous functional implant and have not addressed the high degree of topographical organisation of cells and extracellular matrix constituents within the native osteochondral environment $[27,43,54]$. A further problem is insufficient nutrient transport to the centre of a construct, resulting in reduced cell proliferation or non-optimal conditions for cartilage matrix production $[18,22,33]$. This in turn leads to inhomogeneous tissue formation, with the bulk of cartilage matrix in the periphery and little in the centre [28]. Therefore a recurrent issue in tissue engineering is that constructs lack the mechanical functionality required for clinical application $[21,29]$.

Recent advances in both computational topology design (CTD) and solid free-form fabrication (SFF), or rapid prototyping, provide the opportunity to create more physiologically realistic grafts with controlled architecture $[19,25,26,53]$. Different cell types can be embedded at predefined locations inside the printed polymers [12], allowing the creation of artificial tissues and organs [30,45,49]. The increased control over the scaffold structure also allows strands to be printed closer together, contributing to the mitigation of nutrient limitation.

While current experimental practice relies to a large extent on trial and error, mathematical modelling is ideally suited to improve the quantitative understanding of nutrient transport and could be a key asset in a better controlled design process of tissue engineered constructs. Prompted by this prospect, recent years have seen a rapid increase in the number of mathematical models aimed at the regeneration of articular cartilage $[9,11,14,22,33,34]$; overview in [41]. Mathematical modelling in tissue engineering broadly divides into structural and nutrient approaches, and few authors have considered the interaction between the two. Models that incorporate the effect of the microscopic structure have mainly focused on mechanical quantities, such as the fluid shear stress on the cell membrane as a result of medium perfusion [35,37], or the local mechanical stresses experienced by individual cells as a result of a global mechanical load applied at the macroscopic tissue level [6]. For regular scaffolds, models have been used to describe the mechanoregulation of tissue differentiation and bone formation, using either full simulation or representative volume and homogenization approaches $[1,7,39]$. [15] includes both perfusion and oxygen transport for a specific structure.

In contrast, most previous transport models consider the tissue construct as a homogeneous, continuum material and use parameters that are either empirical or based on rough analytical estimates. Nutrient availability is determined by the relative importance of two key factors: delivery to the cells (through advective and diffusive transport) and cellular utilization. The most important solutes in this respect are oxygen and glucose. In addition, it is necessary to evaluate the production of waste products such as lactate, in order to prevent accumulation. While cellular utilization is simply the product of the per-cell uptake and cell density, this per cell uptake can vary widely and is subject to a highly complex process of metabolic regulation, governed by the levels of the different metabolites and the cellular envi- 
ronment. Models for metabolic networks are being developed to support fundamental biological research [10]. However, due to lack of available data, most models that couple cellular uptake to transport in cartilage tissue engineering adopt a far simpler, workable, approach by considering only a single solute assumed to be critical $[9,11,22,33,34]$, or alternatively, couple glucose, oxygen and lactate using a simple mass balance [42]. On the other hand, in related tissues such as the intervertebral disk, models have been based on actual, empirically measured, coupled relationships [31].

The objective of the present study is to relate microscopic structural and cellular parameters to macroscopic transport and nutrient availability in an organised cartilage construct. This enables the identification of the critical parameters and their interrelationships, thereby informing future cartilage construct design. The asymptotic homogenization technique exploits the regular structure of a scaffold to determine the continuum equations describing fluid, nutrient and waste product transport on the scale of the construct. The homogenization methods provide a rigorous basis for the "bulk averaging" methods that are usually employed to describe these phenomena. Unlike some situations, this rigorous approach does not generate different models from those usually exploited. In fact, the continuum models are those of Darcy flow and advection/diffusion/reaction equations, however the homogenization identifies how the coefficients in these models depend on the micro-scale structure and geometry. To the best of our knowledge, homogenization has not previously been used to characterize 3D construct properties, and so the approach taken here is novel.

\section{Model Setup}

For printed tissue engineering, cartilage cells are extracted from the patient and seeded in a hydrogel. This hydrogel-cell combination is then printed into a regular 3-dimensional structure representative of cartilage tissue in the body. This structure is referred to as a scaffold and provides a template for tissue growth. The scaffold is placed in a construct (the scaffold takes up the entire construct domain) and

(a)

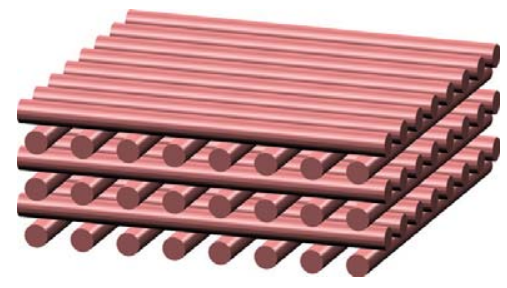

(c)

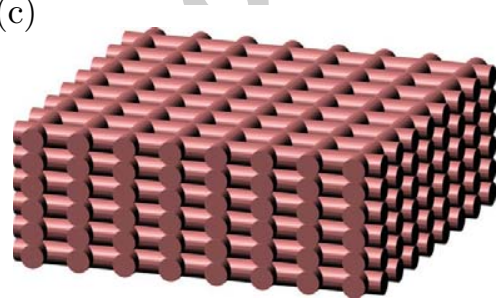

(b)
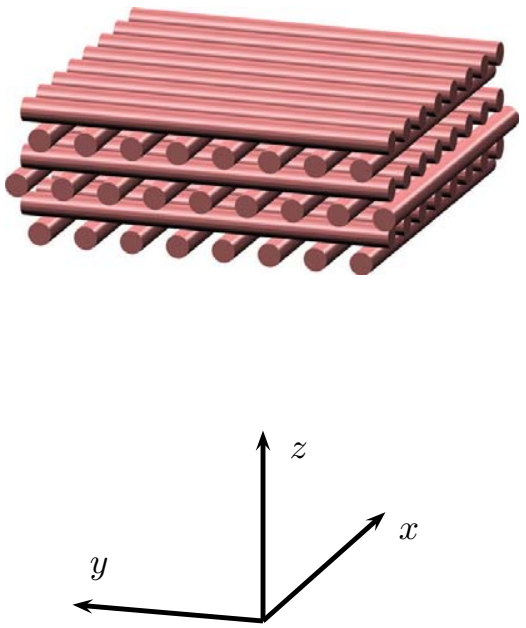

Fig. 1 Depictions of the three configurations of strands: (a) 'overlayed strands', (b) 'offset strands', (c) the 'waffle structure'. 
pumped with culture medium to provide the nutrients required for growth, and to eliminate harmful waste products. The direction of this pumping is flexible, and will be considered as part of the modelling process. The expanding cell population remains inside the gel, and so there are no concerns related to the shear stresses exerted by the flowing medium on the cells.

In this paper we consider three typical printed scaffold configurations, as depicted in Figure 1. The pink regions are the strands containing the cells, and the culture medium flows in the surrounding areas. In each case we assume the strands are cylindrical tubes. The first structure ('overlayed strands') is shown in Figure 1a and is formed from layers of parallel fibres, with the strands in each layer orthogonal to those in the layer below. The same is true of the second structure ('offset strands'); the difference between the two is that in the first the fibres in layer $i$ are placed directly above those in layer $i-2$, while in the second case the $x$-directional fibres are arranged so that those in layer $i$ are placed above the gap in layer $i-2$. The third case (the 'waffle structure') is an adaptation of the first, designed to give enhanced structural integrity. In this instance the configuration is formed by removing the gaps beneath each layer in the overlayed-strands lattice.

The typical construct depth is denoted $h$ and is $\approx 2 \times 10^{-3} \mathrm{~m}$; the typical strand length is denoted $a$ and is $\approx 5 \times 10^{-3} \mathrm{~m}$, and the typical strand diameter is denoted $d$ and is $\approx 2 \times 10^{-4} \mathrm{~m}$. The construct is therefore an $a \times d \times h$ cuboid of volume $\approx 2 \times 10^{-9} \mathrm{~m}^{3}$. The strand separation $r \approx d$, and on this basis the typical density of strands in a construct is $\approx 31$ strands $\mathrm{m}^{-3}$.

We consider the hydrogel and culture medium regions independently. The hydrogel contains the cells that consume nutrients and produce waste products. We simplify the system by assuming a constant cell density in the strands and so neglect the fact that the cell population should be expanding. This assumption should be lifted in future work. Oxygen and glucose are considered to be representative nutrients, and lactate as a representative waste product; in particular the consumption rates for oxygen and glucose are assumed to be proportional to their concentrations, whereas the production rate of lactate is constant (i.e. not related to the underlying oxygen, glucose or lactate concentrations). The culture medium flows under a pressure gradient applied across the scaffold, transporting oxygen and glucose to the hydrogel and removing lactate. This transport is reasonably assumed to be through a combination of advection (by the medium) and diffusion. Within the hydrogel, there is no culture medium flow and so transport is through diffusion alone. The medium has a constant viscosity $\mu$ and so can be modelled as a viscous fluid using the Navier-Stokes equations.

We denote the construct domain by $\Omega \subset \mathcal{R}^{3}$, composed of the hydrogel and medium regions $\Omega_{s}$ and $\Omega_{m}$ respectively; this is depicted in Figure 2 for clarity. The interface between the two regions is denoted by $\Gamma=\partial \Omega_{s} \cap \partial \Omega_{m}$. Typical parameter values for the equations that follow are given in Tables 1 and 2 .

(a)

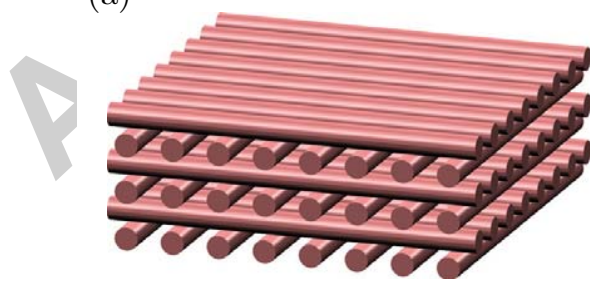

(b)

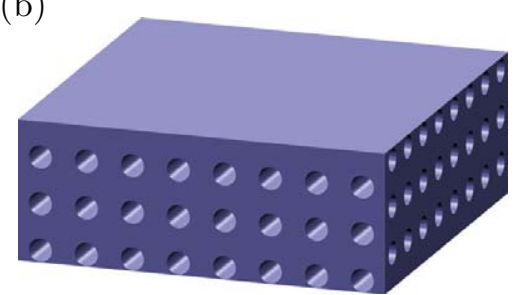

Fig. 2 Schematic of the domain for the 'overlayed strands' configuration showing a) the hydrogel region $\Omega_{s}$ and b) the medium region $\Omega_{m}$ (and $\Omega=\Omega_{s} \cup \Omega_{m}$ ). 


\begin{tabular}{|c|c|c|c|c|}
\hline Parameter & Value & Unit & Description & Reference \\
\hline$d$ & $2 \times 10^{-4}$ & $\mathrm{~m}$ & $\begin{array}{l}\text { typical strand } \\
\text { diameter }\end{array}$ & {$[12]$} \\
\hline$a$ & $5 \times 10^{-3}$ & $\mathrm{~m}$ & $\begin{array}{l}\text { typical strand } \\
\text { length }\end{array}$ & {$[12]$} \\
\hline$h$ & $2 \times 10^{-3}$ & $\mathrm{~m}$ & $\begin{array}{l}\text { typical construct } \\
\text { depth }\end{array}$ & {$[12]$} \\
\hline$\rho$ & $10^{3}$ & $\mathrm{~kg} \mathrm{~m}^{-3}$ & fluid density & {$[51,37,46]$} \\
\hline$\mu$ & $10^{-3}$ & $\mathrm{~kg} \mathrm{~m}^{-1} \mathrm{~s}^{-1}$ & fluid viscosity & {$[51,37,46]$} \\
\hline$D_{m}$ & $3 \times 10^{-9}$ & $\mathrm{~m}^{2} \mathrm{~s}^{-1}$ & $\begin{array}{l}\text { oxygen diffusion } \\
\text { coefficient in fluid }\end{array}$ & {$[16,23]$} \\
\hline$D_{s}$ & $2.6 \times 10^{-9}$ & $\mathrm{~m}^{2} \mathrm{~s}^{-1}$ & $\begin{array}{l}\text { oxygen diffusion } \\
\text { coefficient in gel }\end{array}$ & {$[24,36,8]$} \\
\hline$K_{m}$ & $0.92 \times 10^{-9}$ & $\mathrm{~m}^{2} \mathrm{~s}^{-1}$ & $\begin{array}{l}\text { glucose diffusion } \\
\text { coefficient in fluid }\end{array}$ & {$[2,48]$} \\
\hline$K_{s}$ & $0.78 \times 10^{-9}$ & $\mathrm{~m}^{2} \mathrm{~s}^{-1}$ & $\begin{array}{l}\text { glucose diffusion } \\
\text { coefficient in gel }\end{array}$ & {$[40]$} \\
\hline $\mathcal{D}_{m}$ & $1.4 \times 10^{-9}$ & $\mathrm{~m}^{2} \mathrm{~s}^{-1}$ & $\begin{array}{l}\text { lactate diffusion } \\
\text { coefficient in fluid }\end{array}$ & {$[20]$} \\
\hline $\mathcal{D}_{s}$ & $1.2 \times 10^{-9}$ & $\mathrm{~m}^{2} \mathrm{~s}^{-1}$ & $\begin{array}{l}\text { lactate diffusion } \\
\text { coefficient in gel }\end{array}$ & {$[40]$} \\
\hline
\end{tabular}

Table 1 Typical parameter values.

\begin{tabular}{|c|c|c|c|c|c|}
\hline$\phi$ & $\begin{array}{l}k_{c} \\
\left(\text { cells m}{ }^{-3}\right)\end{array}$ & $\begin{array}{l}k_{g} \\
\left(\mathrm{~m}^{3} \mathrm{~s}^{-1} \mathrm{cell}^{-1}\right)\end{array}$ & $\begin{array}{l}\eta \\
\left(\mathrm{m}^{3} \mathrm{~s}^{-1} \mathrm{cell}^{-1}\right)\end{array}$ & $\begin{array}{l}\text { Remarks } \\
\left(\mathrm{mol} \mathrm{s}^{-1} \text { cell }^{-1}\right)\end{array}$ & Reference \\
\hline $\begin{array}{l}0.2 \times 10^{14}- \\
4.8 \times 10^{14}\end{array}$ & $0.16 \times 10^{-16}$ & - & - & $\begin{array}{l}\text { Bovine } \\
\text { articular } \\
\text { chondrocytes, }\end{array}$ & {$[24]$} \\
\hline $5 \times 10^{12}$ & $10^{-16}$ & $1.4 \times 10^{-17}$ & & $\begin{array}{l}\text { Rabbit } \\
\text { articular } \\
\text { chondrocytes, } \\
\text { monolayer }\end{array}$ & {$[47]$} \\
\hline $1.5 \times 10^{14}$ & $3.6 \times 10^{-18}$ & $1.8 \times 10^{-18}$ & & $\begin{array}{l}\text { Neonatal rat } \\
\text { cardiomyocytes } \\
\text { cultured on } 3 \mathrm{D} \\
\text { scaffolds }\end{array}$ & {$[36]$} \\
\hline $5 \times 10^{11}$ & $1.2 \times 10^{-17}$ & $1.2 \times 10^{-18}$ & $2.64 \times 10^{-17}$ & $\begin{array}{l}\text { Bovine } \\
\text { articular } \\
\text { chondrocytes, } \\
\text { 3D culture }\end{array}$ & {$[32]$} \\
\hline
\end{tabular}

Table 2 Cell density $\phi$, oxygen and glucose consumption rates $k_{c}, k_{g}$, and constant lactate production rate $\eta$. The values from [32] are used as indicative values in the remainder of the analysis.

The flow of the medium in $\Omega_{m}$ is described by the Navier-Stokes equations,

$$
\begin{aligned}
\nabla \cdot \mathbf{u} & =0 \text { in } \Omega_{m} \\
\rho\left(\frac{\partial \mathbf{u}}{\partial t}+(\mathbf{u} \cdot \nabla) \mathbf{u}\right) & =-\nabla p+\mu \nabla^{2} \mathbf{u} \text { in } \Omega_{m} \\
\mathbf{u} & =\mathbf{0} \text { on } \Gamma
\end{aligned}
$$

where $\mathbf{u}=(u, v, w), p, \rho$ and $\mu$ denote the medium velocity, pressure, density and viscosity respectively. The conventional no slip boundary condition (3) is imposed on the medium-scaffold interface. Throughout the whole of $\Omega$, we denote the oxygen, glucose and lactate concentrations by $c, g$ and $l$ respectively. 
We will need to distinguish between these quantities in $\Omega_{m}$ or $\Omega_{s}$ and so let

$$
c_{m}=\left.c\right|_{\Omega_{m}}, c_{s}=\left.c\right|_{\Omega_{s}}, g_{m}=\left.g\right|_{\Omega_{m}}, g_{s}=\left.g\right|_{\Omega_{s}}, l_{m}=\left.l\right|_{\Omega_{m}}, l_{s}=\left.l\right|_{\Omega_{s}} .
$$

The oxygen and glucose transport problems are described by

$$
\begin{array}{rc}
\frac{\partial c_{m}}{\partial t}+\mathbf{u} \cdot \nabla c_{m}=D_{m} \nabla^{2} c_{m} \text { and } \frac{\partial g_{m}}{\partial t}+\mathbf{u} \cdot \nabla g_{m}=K_{m} \nabla^{2} g_{m} \text { in } \Omega_{m} \\
\frac{\partial c_{s}}{\partial t}=D_{s} \nabla^{2} c_{s}-k_{c} \phi c_{s} \text { and } & \frac{\partial g_{s}}{\partial t}=K_{s} \nabla^{2} g_{s}-k_{g} \phi g_{s} \text { in } \Omega_{s} \\
c_{m}=c_{s} \text { and } & g_{m}=g_{s} \text { on } \Gamma \\
D_{m} \frac{\partial c_{m}}{\partial n}=D_{s} \frac{\partial c_{s}}{\partial n} \text { and } & K_{m} \frac{\partial g_{m}}{\partial n}=K_{s} \frac{\partial g_{s}}{\partial n} \text { on } \Gamma .
\end{array}
$$

Equations (5) describe the transport of oxygen and glucose by advection and diffusion (with constant diffusion coefficients $D_{m}$ and $K_{m}$ respectively, measured in units of $\mathrm{m}^{2} \mathrm{~s}^{-1}$ ) in $\Omega_{m}$. In the scaffold, oxygen and glucose diffuse (with constant coefficients $D_{s}$ and $K_{s}$ respectively) and are consumed by the cells, as shown by equations (6). This consumption rate is assumed to be proportional to the respective concentrations, and so the consumption coefficients are given by $\phi k_{c}$ and $\phi k_{g}$ for oxygen and glucose respectively, where $\phi$ is the cell density with units of cells $\mathrm{m}^{-3}$, and $k_{c}, k_{g}$ have units $\mathrm{m}^{2} \mathrm{~mol} \mathrm{~s}^{-1}$ cell $^{-1}$. The use of linear consumption rates as compared to full Michaelis-Menten kinetics has been tested against experimental data in [22] and recommended for the typical concentration ranges observed in constructs. Finally, the boundary conditions on the medium-scaffold interface are given by equations (7) and (8), and correspond to continuity of concentration and flux.

The lactate transport problem is described similarly by

$$
\begin{aligned}
\frac{\partial l_{m}}{\partial t}+\mathbf{u} \cdot \nabla l_{m} & =\mathcal{D}_{m} \nabla^{2} l_{m} \text { in } \Omega_{m}, \\
\frac{\partial l_{s}}{\partial t} & =\mathcal{D}_{s} \nabla^{2} l_{s}+\eta \phi \text { in } \Omega_{s}, \\
l_{m} & =l_{s} \text { on } \Gamma, \\
\mathcal{D}_{m} \frac{\partial l_{m}}{\partial n} & =\mathcal{D}_{s} \frac{\partial l_{s}}{\partial n} \text { on } \Gamma,
\end{aligned}
$$

where $\mathcal{D}_{m}$ and $\mathcal{D}_{s}$ are the constant lactate diffusion coefficients in $\Omega_{m}$ and $\Omega_{s}$ respectively. The product $\eta \phi$ is the constant lactate production rate; $\eta$ has units $\mathrm{m}^{3} \mathrm{~mol} \mathrm{~s}^{-1}$ cell ${ }^{-1}$, and can be written in the form $\eta=[l] k_{l}$ to compare directly with the oxygen and glucose consumption terms, where $[l]$ is a typical lactate concentration, measured in units of $\mathrm{mol} \mathrm{m}^{-3}$, and $k_{l}$ is a rate constant (comparable to $k_{c}$ and $k_{g}$ ) with units $\mathrm{m}^{3} \mathrm{~s}^{-1}$ cell ${ }^{-1}$. This formulation is necessary due to the assumption of a constant production rate for lactate, and to interpret the lactate production data available in the literature.

In reality, the production of lactate depends on the amount of oxygen and glucose consumed, and so the transport problems for oxygen, glucose and lactate should be coupled. However, the way in which the nutrients interact is not fully understood for chondrocytes [17,31], and therefore we consider only the decoupled system. This will give insight into the nutrient transport problem, even though it does not model the full biological system.

\section{Dimensional Analysis}

In this Section we perform dimensional analysis of the system given by (1)-(12). This determines the dominant transport processes (advection, diffusion or consumption/production) in the radial direction through the strand (lengthscale $d$ ), and also down the strand length (lengthscale $a$ ). As a consequence, we determine two design criteria that ensure sufficient nutrient is supplied to the cells to meet the demands 
of consumption, and also to ensure that lactate is removed from the construct before it reaches toxic levels.

A summary of the timescales for advection, diffusion and consumption/production in each direction is given in Table 3, calculated using the values from [32] in Table 2. There are no clear data on the typical medium flow velocities (a pressure gradient is applied across the construct to generate the fluid flow; however, this gradient is not measured experimentally, and so is unknown here) therefore we scale the fluid velocity $\mathbf{u}$ with an unknown $U$ and determine a minimum value for $U$ that ensures lactate removal from the construct. The cells in the hydrogel are not in contact with the culture medium and so there is no shear stress exerted on the cells by the medium flow. As a consequence, we do not calculate a maximum bound on $U$ (the only bound necessary would be to ensure the structural stability of the scaffold).

Oxygen and glucose are consumed by the cells in the hydrogel, and reach these cells from the culture medium by diffusion through the hydrogel. Assuming that sufficient nutrient is supplied in the culture medium, the strand diameter must be small enough to ensure that sufficient oxygen/glucose is supplied to all cells to meet the demands of consumption. Using the results from Table 3, it is clear that the timescale for nutrient diffusion dominates over that for nutrient consumption in the strand $\left(15.4 \mathrm{~s} \ll 1.67 \times 10^{5} \mathrm{~s}\right.$ for oxygen and $51.3 \mathrm{~s} \ll 1.67 \times 10^{6} \mathrm{~s}$ for glucose) and so the growth of the cells in the hydrogel will

\begin{tabular}{|c|c|c|}
\hline Description & Formula & Timescale (secs) \\
\hline $\begin{array}{l}\text { Advection in direction of } \\
\text { strand length }\end{array}$ & $a / U$ & $\frac{5}{U} \times 10^{-3}$ \\
\hline $\begin{array}{l}\text { Oxygen diffusion down } \\
\text { strand }\end{array}$ & $a^{2} / D_{s}$ & $9.6 \times 10^{3}$ \\
\hline $\begin{array}{l}\text { Oxygen diffusion } \\
\text { radially in strand }\end{array}$ & $d^{2} / D_{s}$ & 15.4 \\
\hline $\begin{array}{l}\text { Oxygen diffusion in } \\
\text { medium in direction of } \\
\text { strand length }\end{array}$ & $a^{2} / D_{m}$ & $8.3 \times 10^{3}$ \\
\hline $\begin{array}{l}\text { Oxygen diffusion } \\
\text { radially in medium }\end{array}$ & $d^{2} / D_{m}$ & 13.3 \\
\hline $\begin{array}{l}\text { Oxygen consumption in } \\
\text { strand }\end{array}$ & $1 /\left(k_{c} \phi\right)$ & $1.67 \times 10^{5}$ \\
\hline $\begin{array}{l}\text { Glucose diffusion down } \\
\text { strand }\end{array}$ & $a^{2} / K_{s}$ & $3.2 \times 10^{4}$ \\
\hline $\begin{array}{l}\text { Glucose diffusion } \\
\text { radially in strand }\end{array}$ & & 51.3 \\
\hline $\begin{array}{l}\text { Glucose diffusion in } \\
\text { medium in direction of } \\
\text { strand length }\end{array}$ & $a^{2} / K_{m}$ & $2.7 \times 10^{4}$ \\
\hline $\begin{array}{l}\text { Glucose diffusion } \\
\text { radially in medium }\end{array}$ & $d^{2} / K_{m}$ & 43.5 \\
\hline $\begin{array}{l}\text { Glucose consumption in } \\
\text { strand }\end{array}$ & $1 /\left(k_{g} \phi\right)$ & $1.67 \times 10^{6}$ \\
\hline $\begin{array}{l}\text { Lactate diffusion down } \\
\text { strand }\end{array}$ & $a^{2} / \mathcal{D}_{s}$ & $2.08 \times 10^{4}$ \\
\hline $\begin{array}{l}\text { Lactate diffusion } \\
\text { radially in strand }\end{array}$ & $d^{2} / \mathcal{D}_{s}$ & 33.3 \\
\hline $\begin{array}{l}\text { Lactate diffusion in } \\
\text { medium in direction of } \\
\text { strand length }\end{array}$ & $a^{2} / \mathcal{D}_{m}$ & $1.79 \times 10^{4}$ \\
\hline $\begin{array}{l}\text { Lactate diffusion } \\
\text { radially in medium }\end{array}$ & $d^{2} / \mathcal{D}_{m}$ & 28.6 \\
\hline $\begin{array}{l}\text { Lactate production in } \\
\text { strand }\end{array}$ & $1 /\left(k_{l} \phi\right)$ & $3 \times 10^{4}$ \\
\hline
\end{tabular}

Table 3 Transport timescales. 
not be impeded by a lack of nutrient. This will always be the case unless the diffusive and consumption timescales in the strand are of the same order of magnitude (then growth of cells would be diffusion limited). This occurs when the strand diameter $d$ increases to a critical value, $d=d_{\max }$ determined by

$$
\frac{d_{\max }^{2}}{D_{s}}=\frac{1}{k_{c} \phi} \text { for oxygen and } \frac{d_{\max }^{2}}{K_{s}}=\frac{1}{k_{g} \phi} \text { for glucose. }
$$

This formula determines $d_{\max }$ as a function of the cell density $\phi$ for fixed $k_{c}, D_{s}, k_{g}$ and $K_{s}$,

$$
d_{\max }=\min \left\{\sqrt{\frac{D_{s}}{k_{c} \phi}}, \sqrt{\frac{K_{s}}{k_{g} \phi}}\right\},
$$

and adhering to this maximum value for the strand diameter will ensure that $c_{s}$ and $g_{s}$ never reach zero.

Lactate is produced in the strand as a waste product of respiration, and will be toxic to the cells above a critical concentration, $l^{*}$ say. It is crucial that lactate is eliminated from the construct before this critical concentration $l^{*}$ is reached. In [50], the authors estimate the concentration of lactic acid that would be harmful to the growing cells for a buffered culture medium. It is assumed that growing cells can tolerate a $\mathrm{pH}$ change of up to \pm 0.5 , and titration experiments indicated that the harmful concentration of lactic acid in a buffered medium was $\approx 0.4 \mathrm{~mol} \mathrm{~m}^{-3}$. Lactic acid is produced from the dissociation of lactate (in equal molar ratios) and so we take $l^{*}=0.4 \mathrm{~mol} \mathrm{~m}^{-3}$ as an indicative value. We also use $l^{*}$ as a concentration scale to determine $k_{l}$ from $\eta\left(k_{l}=\eta / l^{*}\right)$; this gives $k_{l}=6.6 \times 10^{-17} \mathrm{~m}^{3} \mathrm{~s}^{-1}$ cell $^{-1}$.

Once the lactate is produced in the strand, it is transported to the culture medium by diffusion. Therefore, the timescale for lactate production in a strand must be longer than that for lactate diffusion in a strand,

$$
\frac{1}{k_{l} \phi} \geq \frac{d^{2}}{\mathcal{D}_{s}}
$$

This gives a second formulation for $d_{\max }$, determined by

$$
d_{\max }=\sqrt{\frac{\mathcal{D}_{s}}{k_{l} \phi}} .
$$

For the parameter values above, this yields

$$
d_{\max }=\frac{4.2 \times 10^{4}}{\sqrt{\phi}} .
$$

Combining the results for oxygen, glucose and lactate, $d_{\max }$ must satisfy

$$
d_{\max }=\min \left\{\sqrt{\frac{D_{s}}{k_{c} \phi}}, \sqrt{\frac{K_{s}}{k_{g} \phi}}, \sqrt{\frac{\mathcal{D}_{s}}{k_{l} \phi}}\right\},
$$

and this represents the first design criterion for the construct.

Once the lactate has diffused into the culture medium, it must be removed from the construct to eliminate the possibility of it resulting in cell death at later times (e.g. by diffusion back into the strands). As shown in Table 3 , the timescales for lactate production $\left(1 / k_{l} \phi\right)$ and lactate diffusion in the direction of the strand length $\left(a^{2} / \mathcal{D}_{m}\right)$ are of the same order of magnitude $\left(3 \times 10^{4}\right.$ secs and $1.79 \times 10^{4}$ secs, respectively). Therefore, we choose the minimum perfusion velocity $U_{\min }$ as that which causes the timescale for advection $(a / U)$ to balance that for lactate production. Then for $U>U_{m i n}$, advection will aid lactate removal from the construct. This balancing yields

$$
U_{\min }=a k_{l} \phi .
$$

Based on a typical value $a=5 \times 10^{-3} \mathrm{~m}$ from Table 1 , with $\phi=5 \times 10^{11}$ cells $\mathrm{m}^{-3}, k_{l}=6.6 \times$ $10^{-17} \mathrm{~m}^{3} \mathrm{~s}^{-1}$ cell $^{-1}$,

$$
U_{\text {min }}=a k_{l} \phi=1.65 \times 10^{-7} \mathrm{~m} \mathrm{~s}^{-1}
$$

is the minimum velocity required to remove lactate from the construct. As expected, the timescale for advection is then $\mathcal{O}\left(10^{4}\right)$ secs, i.e. the same order of magnitude as the diffusion and production timescales. This $U_{\min }$ is the second design criterion for the construct. 


\section{Homogenization Analysis for the Culture Medium}

The dimensional analysis described in Section 3 determined two design criteria for the tissue engineering construct, based on transport considerations for a single strand. In this Section we extend this by analysing the dependence of the continuum perfusion properties of the multi-strand scaffold on the micro-structure. In particular, this determines which of the three proposed structures in Figure 1 is optimal, and the preferable perfusion direction of the culture medium.

The link between the perfusion properties and micro-structure is determined through asymptotic homogenization, by exploiting the fact that the strand separation ( $r$ say) is much smaller than the construct dimensions, so that

$$
\epsilon:=\frac{r}{a} \ll 1
$$

The two length scales characterized by $r$ (the micro-scale) and $a$ (the macro- or construct scale) are therefore well separated, so given the flow system on the micro-scale described by (1)-(3), it is possible to use asymptotics to move between the micro- and macro- descriptions and derive the continuum flow equations on the construct scale. The success of this approach depends on the micro-structure being periodic; we denote the periodic domain by $V$, composed of the strand region $V_{s}$ and medium region $V_{m}$. The details of this homogenization process are included in Appendix A so as not to cloud the purpose of this paper.

The homogenization returns Darcy's law for the effective medium velocity on the construct scale, given in non-dimensional form by

$$
\langle\mathbf{u}\rangle=-\kappa \nabla p
$$

where

$$
\boldsymbol{\nabla} \cdot\langle\mathbf{u}\rangle=0 .
$$

Here $\langle\mathbf{u}\rangle$ denotes the averaged medium velocity. The symbol $\boldsymbol{\kappa}$ represents the (non-dimensional) permeability tensor and describes the dependence of $\langle\mathbf{u}\rangle$ on the micro-scale properties. The components of $\boldsymbol{\kappa}$ are given by

$$
\kappa_{i j}=\frac{1}{|V|} \iiint_{V_{m}} w_{i}^{j} \mathrm{~d} V
$$

where $w_{i}^{j}$ describe the variation in the medium velocity on the micro-scale and are determined through a canonical cell problem (69)-(71) (see Appendix A for further details). The key advantage of this approach is that it is not necessary to prescribe the micro-scale geometry to perform the homogenization, and so it is possible to investigate the impact of geometry on the perfusion properties.

The solid fraction $n_{s}$ of each structure is defined by

$$
n_{s}=\frac{\left|V_{s}\right|}{|V|} .
$$

This is calculable for each of the three structures in Figure 1. For the overlayed and offset strand structures (Figures 1a and b),

$$
n_{s}=\frac{\pi d}{4 r}
$$

whereas for the waffle structure (Figure 1c)

$$
n_{s}=\frac{\pi d}{2 r}-\frac{2 d^{2}}{3 r^{2}}
$$

While the shape of each structure is defined, there is a degree of freedom available by choosing the separation distance $r$ between the centres of adjacent strands, relative to the strand diameter $d$. We thus identify the geometric parameter $\lambda=(r-d) / d$, which in theory varies between zero (if adjacent strands are in contact), and infinity (if the strands are infinitesimally thin compared with their separation). In 


\section{ACCEPTED MANUSCRIPT}

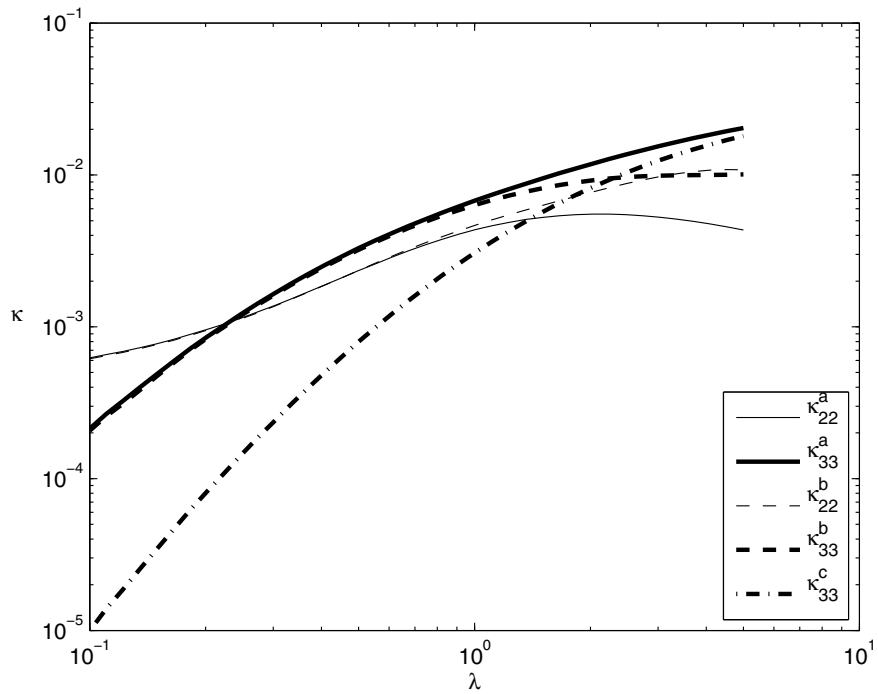

Fig. 3 Graph of the permeability values for the three structures (a)-(c) depicted in Figure 1.

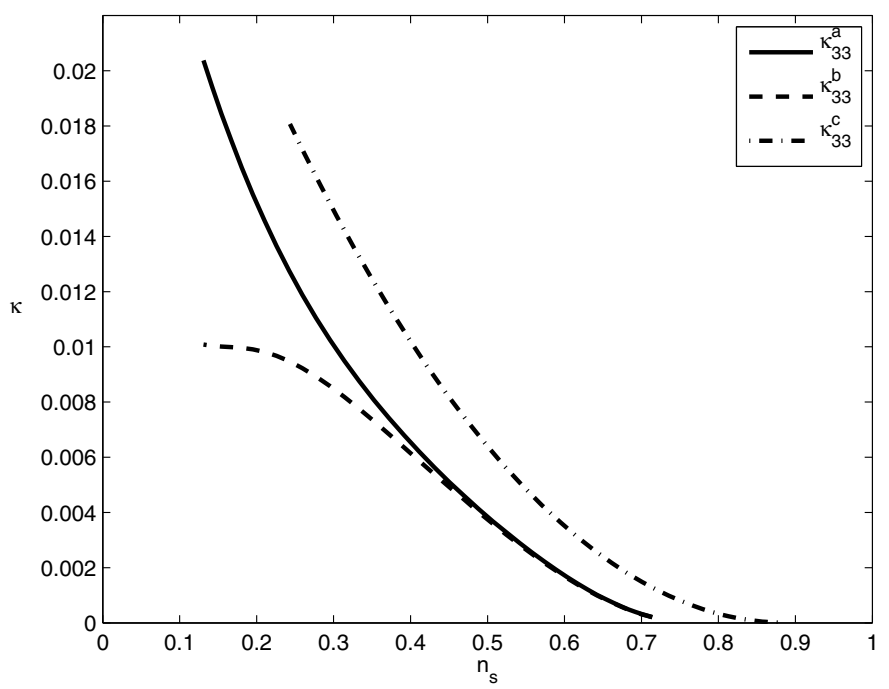

Fig. 4 Graph of the vertical permeability for the three structures (a)-(c) of Figure 1 as a function of the solid fraction $n_{s}$.

this investigation we will consider values of $\lambda$ in the physiologically realistic range $[0.1,5]$. For example, if the strand separation is double the strand diameter, $r=2 d$ and $\lambda=1$.

The dimensionless permeability $\boldsymbol{\kappa}$ only depends on $r$ and $d$ through the parameter $\lambda$, because all lengths on the micro-scale are non-dimensionalised with $r$. To obtain the dimensional permeability tensor, we take the constitutive law (22) and write each quantity in terms of the dimensional form. This gives 
Darcy's equation in the form

$$
\langle\mathbf{u}\rangle=-\frac{1}{\mu} \boldsymbol{\kappa}^{\operatorname{dim}} \cdot \nabla p
$$

where each term is now a dimensional quantity and the permeability is given by

$$
\kappa^{\operatorname{dim}}=r^{2} \kappa(\lambda)
$$

This corresponds to the result that the permeability decreases as the mesh becomes ever finer.

The periodic cell problem (69)-(71) was solved for each structure, and for a range of values of $\lambda$, using the finite elements package 'COMSOL Multiphysics'" The (dimensionless) permeability tensors were then calculated using the definition (24). The tensors obtained were all diagonal as the strands are only positioned in the co-ordinate directions, and the components are logarithmically plotted in Figure 3 as a function of the geometric parameter $\lambda$.

We note (where the superscript refers to the structure thus defined in Figure 1) that $\kappa_{11}^{a}=\kappa_{22}^{a}$ by symmetry, and that $\kappa_{11}^{c}=\kappa_{22}^{c}=0$ (i.e. the waffle structure is impermeable laterally). Furthermore, calculations show that $\kappa_{11}^{b}$ is almost indistinguishable from $\kappa_{11}^{a}$ over this range of $\lambda$.

The results obtained can aid the optimisation of the design of the tissue engineering construct. The aim, with all other factors being equal, is for a higher permeability since the medium velocity will consequently be higher for a given pressure gradient, by (28), leading to an increased lactate removal rate. This assumes that the increased velocity will not damage the structural integrity of the scaffold.

Figure 3 shows that for most values of $\lambda$, the permeability for flow in the $z$-direction $\left(\kappa_{33}\right)$ is greater than the lateral permeability $\left(\kappa_{11}, \kappa_{22}\right)$. This result is reversed if $\lambda \ll 1$ in the overlayed or offset strands structure. We also note that, curiously, the permeability of flow in the $y$-direction through the offset strands structure, $\kappa_{22}^{b}$, is the largest permeability component in that structure for $\lambda \approx 5$ (although the value of $\kappa_{33}^{b}$ is only slightly lower).

Thus, for realistic, namely $\mathcal{O}(1)$, values of $\lambda$ it is optimal to perfuse the medium through each scaffold vertically (i.e. in the $z$-direction of Figure 1). Of course, for the waffle structure this is the only option.

Secondly, we consider which of the three structures is the best choice for the construct. Figure 3 suggests that for each value of $\lambda$, the overlayed-strands structure has the greatest vertical permeability, and should thus be favoured. However, $\lambda$ is not the most physically-relevant parameter here. Rather, the solid fraction $n_{s}$, which governs the number of cells which may be accommodated in the scaffold, is a more useful measure. Using (26)-(27), a 1-1 relationship between $n_{s}$ and $\lambda$ may be identified for each structure; this is used to display the vertical permeabilities of each structure as a function of $n_{s}$ in Figure 4. This graph shows that the waffle structure is clearly the most desirable configuration given that the vertical permeability is higher for each value of $n_{s}$.

In conclusion, two further design criteria for the construct are to use the waffle structure for the scaffold, and to perfuse the medium through the scaffold vertically.

\section{Homogenization Analysis for Oxygen, Glucose and Lactate Transport}

Homogenization can also be applied to the oxygen, glucose and lactate transport problems to determine the dependence of the construct-scale transport properties on the micro-scale. The process returns advection-diffusion-reaction (where reaction incorporates consumption or production) equations for the oxygen, glucose and lactate concentrations, and in particular determines the dependence of the construct-scale advection, diffusion and consumption/production coefficients on the micro-scale transport coefficients and geometry. The detail of the homogenization process is provided in Appendix B.

\footnotetext{
${ }^{1}$ Developed and distributed by COMSOL Inc. Full details available online at http://www.comsol.com/.
} 
The non-dimensional construct-scale transport equations are given by

$$
\begin{aligned}
\operatorname{Pe}_{G}^{c}\left(\frac{\partial\langle c\rangle}{\partial t}+\tilde{\mathbf{u}}_{c} \cdot \nabla\langle c\rangle\right) & =\boldsymbol{\nabla} \cdot\left(\underline{\underline{\boldsymbol{\Omega}}}^{c} \boldsymbol{\nabla}\langle c\rangle\right)-\mathrm{Da}_{G}^{c} n_{s}\langle c\rangle, \\
\mathrm{Pe}_{G}^{g}\left(\frac{\partial\langle g\rangle}{\partial t}+\tilde{\mathbf{u}}_{g} \cdot \boldsymbol{\nabla}\langle g\rangle\right) & =\boldsymbol{\nabla} \cdot\left(\underline{\underline{\boldsymbol{\Omega}}}^{g} \boldsymbol{\nabla}\langle g\rangle\right)-\mathrm{Da}_{G}^{g} n_{s}\langle g\rangle, \\
\operatorname{Pe}_{G}^{l}\left(\frac{\partial\langle l\rangle}{\partial t}+\tilde{\mathbf{u}}_{l} \cdot \boldsymbol{\nabla}\langle l\rangle\right) & =\boldsymbol{\nabla} \cdot\left(\underline{\underline{\boldsymbol{\Omega}}}^{l} \boldsymbol{\nabla}\langle l\rangle\right)+\mathrm{Da}_{G}^{l} n_{s} .
\end{aligned}
$$

The superscripts $c, g$ or $l$ identify a coefficient with oxygen, glucose or lactate respectively. $\mathrm{Pe}_{G}$ is the construct-scale Péclet number and represents the ratio of diffusive to advective timescales on the construct-scale (e.g. $\left.\mathrm{Pe}_{G}^{c}=U a / D_{m}\right) . \mathrm{Pe}_{G}$ is an $\mathcal{O}(1)$ parameter, indicating that diffusion and advection are of equal importance on the construct-scale. The effective advective and diffusion coefficients are denoted $\tilde{\mathbf{u}}$ and $\underline{\underline{\boldsymbol{\Omega}}}$; for oxygen these are given by

$$
\begin{aligned}
\tilde{\mathbf{u}}_{c} & =\left\langle\mathbf{u}^{(0)}\right\rangle+\frac{1}{|V|} \int_{V_{m}}\left(\boldsymbol{\nabla}_{x} \boldsymbol{\chi}_{m}^{c}\right)^{T} \cdot \mathbf{u}^{(0)} \mathrm{d} V \\
\underline{\boldsymbol{\Omega}}^{c} & =\left(1+n_{s}(\bar{D}-1)\right) \underline{\underline{\mathbf{I}}}+\frac{1}{|V|} \int_{V_{m}}\left(\boldsymbol{\nabla}_{x} \boldsymbol{\chi}_{m}^{c}\right)^{T} \mathrm{~d} V+\frac{1}{|V|} \int_{V_{s}} \bar{D}\left(\boldsymbol{\nabla}_{x} \boldsymbol{\chi}_{s}^{c}\right)^{T} \mathrm{~d} V,
\end{aligned}
$$

where $\boldsymbol{\chi}_{m}^{c}$ and $\boldsymbol{\chi}_{s}^{c}$ are solutions of the cell problem (36)-(40) and $\bar{D}=D_{s} / D_{m}$ is the ratio of oxygen diffusion coefficients (see Appendix B for further details). This definition extends naturally to the glucose and lactate components.

Finally, $\mathrm{Da}_{G}$ is the construct-scale Dämkohler number and represents the ratio of diffusive to consumptive time scales on the construct-scale $\left(\right.$ e.g. $\left.\mathrm{Da}_{G}^{c}=k_{c} a^{2} \phi / D_{m}\right)$. Oxygen and glucose are supplied to the hydrogel by advection and diffusion, which both occur on the same time scale on the construct-scale. The time scale for oxygen or glucose consumption $\left(1 / k_{c} \phi\right.$ or $1 / k_{g} \phi$ respectively) must not be shorter than that for oxygen or glucose diffusion $\left(a^{2} / D_{m}\right.$ or $a^{2} / K_{m}$ respectively) to ensure that consumption does not exceed the available nutrient supply. This translates to the conditions that $\mathrm{Da}_{G}^{c}$ and $\mathrm{Da}_{G}^{g}$ must both be no greater than $\mathcal{O}(1)$. Similarly, lactate is produced in the strands and must be removed from the construct by a combination of diffusion and advection (which occur on the same time scale on the construct-scale). This removal can only be achieved providing the time scale for lactate production $\left(1 / k_{l} \phi\right)$ is not shorter than that for lactate diffusion $\left(a^{2} / \mathcal{D}_{m}\right)$, therefore $\operatorname{Da}_{G}^{l}$ must be no greater than $\mathcal{O}(1)$. In summary, $\mathrm{Da}_{G}$ must be no greater than $\mathcal{O}(1)$ and this provides a maximum bound on the printed cell density $\phi$, given by

$$
\phi_{\max }=\min \left(\frac{D_{m}}{a^{2} k_{c}}, \frac{K_{m}}{a^{2} k_{g}}, \frac{\mathcal{D}_{m}}{a^{2} k_{l}}\right) .
$$

This $\phi_{\max }$ imposes a further design criterion for the scaffold - the maximum printed cell density $\phi$, given chosen values for the rate constants $k_{c}, k_{g}$ and $k_{l}$.

The advection and diffusion components $\tilde{\mathbf{u}}$ and $\underline{\underline{\boldsymbol{\Omega}}}$ may be simplified by exploiting the impact of geometry on the solutions $\boldsymbol{\chi}_{m}$ and $\boldsymbol{\chi}_{s}$. For oxygen, these are determined through the cell problem

$$
\begin{aligned}
\nabla_{x}^{2} \boldsymbol{\chi}_{m}^{c} & =\mathbf{0} \text { in } V_{m}, \\
\nabla_{x}^{2} \boldsymbol{\chi}_{s}^{c} & =\mathbf{0} \text { in } V_{s}, \\
\boldsymbol{\chi}_{m}^{c} & =\boldsymbol{\chi}_{s}^{c} \text { on } \Sigma_{\epsilon}, \\
\left(\boldsymbol{\nabla}_{x} \boldsymbol{\chi}_{m}^{c}\right)^{T} \cdot \mathbf{n}-\bar{D}\left(\boldsymbol{\nabla}_{x} \boldsymbol{\chi}_{s}^{c}\right)^{T} \cdot \mathbf{n} & =(\bar{D}-1) \mathbf{n} \text { on } \Sigma_{\epsilon}, \\
\int_{V_{m}} \boldsymbol{\chi}_{m}^{c} \mathrm{~d} V+\int_{V_{s}} \boldsymbol{\chi}_{s}^{c} \mathrm{~d} V & =0 .
\end{aligned}
$$

For a unit cell with three planes of symmetry (as is the case for each of the geometries that we consider), $\chi_{m, i}^{c}$ will be odd in $x_{i}$ and even in the other two coordinates. Hence the gradients $\partial \chi_{m, i}^{c} / \partial x_{j}$ will be 
even in all coordinates if $i=j$; and odd in $x_{i}, x_{j}$ and even in $x_{k}$ if $i \neq j \neq k \neq i$. However, $u_{i}^{(0)}$ is odd in $x_{i}$ (and even in the other two coordinates), therefore the integrand of the integral term in the definition of $\tilde{\mathbf{u}}$ given by (33) is odd in one coordinate. As such, the integral is zero, and for these types of unit cell geometries, $\tilde{\mathbf{u}}=\left\langle\mathbf{u}^{(0)}\right\rangle$, and so the average oxygen, glucose and lactate concentrations are all advected by the Darcy velocity (22). Given the conclusions of Section 4, the waffle structure shown in Figure 1c with vertical perfusion is optimal. Throughout the analysis that follows, we assume that the scaffold structure is the waffle, with $\kappa_{33}^{c}=5 \times 10^{-3}$ and $n_{s}=\pi / 4-1 / 6$, and that the medium is perfused vertically through the scaffold.

From the result above for the gradient $\partial \chi_{m, i}^{c} / \partial x_{j}$, the effective diffusion coefficient given by (34) will be orthotropic. When $\lambda=1$, the bulk term in (34), given by $\Omega_{b u l k}^{c} \underline{\underline{\mathbf{I}}}=\left(1+n_{s}(\bar{D}-1)\right) \underline{\underline{\mathbf{I}}}$, is equal to $0.92 \underline{\underline{I}}$. The perturbations to this bulk term due to the integral terms in (34) may be calculated using 'COMSOL Multiphysics' and are of magnitude $10^{-3}$ or smaller. Hence, the perturbations are negligible given the bulk result, and it is reasonable to assume that $\underline{\boldsymbol{\Omega}}^{c}$ is approximately isotropic and given by

$$
\underline{\underline{\boldsymbol{\Omega}}}^{c}=\Omega_{b u l k}^{c} \underline{\underline{\mathbf{I}}}=\left(1+n_{s}(\bar{D}-1)\right) \underline{\underline{\mathbf{I}}} .
$$

This result extends naturally to the glucose and lactate problems. As such, for the waffle structure the problem simplifies to a one dimensional system, merely depending on $z$ and $t$ ( $z$ is the vertical coordinate). If we furthermore assume that a steady state has been reached, the decoupled equations (30)-(32) can be solved analytically.

Solving (22) and (23) determines the velocity $\langle\mathbf{u}\rangle$ for the given problem. For the waffle structure with vertical perfusion, this velocity only has a non-zero component in the $z$-direction, given by

$$
\langle w\rangle=\frac{\kappa_{33}^{c} \Delta P}{h},
$$

where $\Delta P$ is the externally applied pressure difference, $h$ is the (dimensionless) height of the construct, and $\kappa_{33}^{c}$ was determined in Section 4 (i.e. the boundary conditions for $p$ are $p(0)=\Delta P, p(h)=0$ ). (30)-(32) can now be solved, in steady-state, to determine the average oxygen, glucose and lactate concentrations as a function of the distance, $z$, along the construct.

We prescribe boundary conditions on the medium inlet and outlet, $z=0$ and $z=h$ respectively. On $z=0$

$$
\langle c\rangle=c_{0},\langle g\rangle=g_{0} \text { and }\langle l\rangle=0,
$$

so that no lactate enters the scaffold through the culture medium. On the outlet $z=h$, we prescribe no diffusive flux of oxygen, glucose or lactate out of the scaffold, so that

$$
\frac{\mathrm{d}\langle c\rangle}{\mathrm{d} z}=\frac{\mathrm{d}\langle g\rangle}{\mathrm{d} z}=\frac{\mathrm{d}\langle l\rangle}{\mathrm{d} z}=0 .
$$

The average oxygen concentration is found to be

$$
\langle c\rangle=c_{0}\left(\frac{\gamma^{-} e^{\left(\gamma^{+} z+\gamma^{-} h\right)}-\gamma^{+} e^{\left(\gamma^{-} z+\gamma^{+} h\right)}}{\gamma^{-} e^{\gamma^{-} h}-\gamma^{+} e^{\gamma^{+} h}}\right),
$$

where $\gamma^{ \pm}$are given by

$$
\gamma^{ \pm}=\frac{\operatorname{Pe}_{G}^{c}\langle w\rangle \pm \sqrt{\left(\mathrm{Pe}_{G}^{c}\langle w\rangle\right)^{2}+4 K^{c} \operatorname{Da}_{G}^{c} n_{s}}}{2 \Omega_{b u l k}^{c}},
$$

The glucose solution takes the same form, replacing parameters with their glucose analogues. The average lactate concentration is given by

$$
\langle l\rangle=\frac{\Omega_{b u l k}^{l} \beta}{\alpha^{2}} e^{-\alpha h / \Omega_{b u l k}^{l}}\left(1-e^{\alpha z / \Omega_{b u l k}^{l}}\right)+\frac{\beta}{\alpha} z,
$$




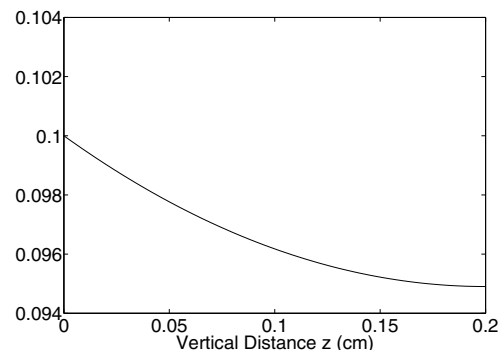

(a) Average Oxygen Concentration $\left(\mathrm{mol} \mathrm{m}{ }^{-3}\right)$

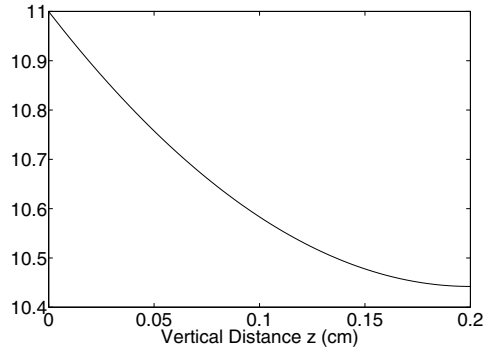

$\langle c\rangle$ (b) Average Glucose Concentration $\langle g\rangle$ $\left(\mathrm{mol} \mathrm{m}^{-3}\right)$

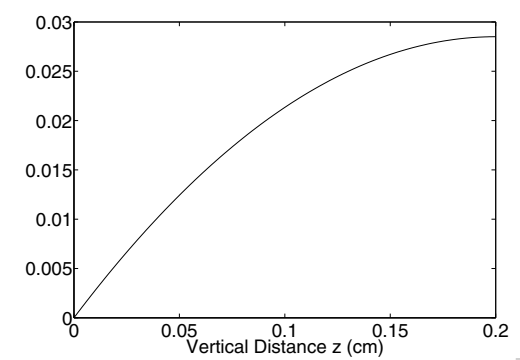

(c) Average Lactate Concentration $\langle l\rangle$ $\left(\mathrm{mol} \mathrm{m}^{-3}\right)$

Fig. 5 Graphs of the (dimensional) average concentrations, plotted as functions of distance up the construct, when $\mathrm{Pe}_{G}^{c}=0.625, \mathrm{Pe}_{G}^{g}=2.03, \mathrm{Pe}_{G}^{l}=0.625, \mathrm{Da}_{G}^{c}=1, \mathrm{Da}_{G}^{g}=1, \mathrm{Da}_{G}^{l}=1.33, \bar{D}=0.87, \bar{K}=0.84, \overline{\mathcal{D}}=0.84$ and $\Delta P=1.4 \times 10^{-5} \mathrm{~N} \mathrm{~m}^{-2}$.

where

$$
\alpha=\operatorname{Pe}_{G}^{l}\langle w\rangle \text { and } \beta=\operatorname{Da}_{G}^{l} n_{s} .
$$

These solutions are plotted in dimensional form in Figure 5 using typical values from Table 1. To re-dimensionalise the solutions requires the choice of concentration scales for oxygen, glucose and lactate. We use typical inlet concentrations for oxygen and glucose of $0.1 \mathrm{~mol} \mathrm{~m}{ }^{-3}$ and $11 \mathrm{~mol} \mathrm{~m}{ }^{-3}$ respectively. For lactate, we use the critical concentration $l^{*}=0.4 \mathrm{~mol} \mathrm{~m}^{-3}$, as discussed previously. We also assume that the construct height is $0.2 \mathrm{~cm}$. As anticipated, the graphs show that $\langle c\rangle$ and $\langle g\rangle$ are at their maximum at the inlet, and decay monotonically with $z$ as the medium passes through the construct and the nutrients are consumed. Similarly, the lactate concentration is zero at the inlet but builds up in the medium as lactate is produced in the cells. For each of the three species, the concentration variations from the inlet conditions are small. It should also be noted that the lactate concentration always remains lower than the critical value of $0.4 \mathrm{~mol} \mathrm{~m}^{-3}$ identified earlier.

As described above, oxygen and glucose are depleted over the length of the construct. Cells require a certain concentration of oxygen, $c_{\min }$ say, to produce ECM successfully, and therefore the inlet oxygen concentration, $c_{0}$, must be maintained at a high enough level that $\langle c\rangle>c_{\min }$ throughout the construct. The oxygen concentration is a minimum at the outlet $z=h$, and so specifying $\langle c\rangle=c_{\min }$ on $z=h$ in (45) yields

$$
c_{0}=c_{\min }\left(\frac{\gamma^{-} e^{\gamma^{-} h}-\gamma^{+} e^{\gamma^{+} h}}{\left(\gamma^{-}-\gamma^{+}\right) e^{\left(\gamma^{+}+\gamma^{-}\right) h}}\right) .
$$

In addition, chondrogenic differentation is limited when $c$ exceeds a maximum value, $c_{\max }$ say. Figure $5 \mathrm{a}$ clearly shows that the average oxygen concentration $\langle c\rangle$ decreases from a maximum value at the inlet, 
and therefore it is appropriate to pick the inlet boundary condition to be this maximum permissible value,

$$
\langle c\rangle=c_{\max } \text { on } z=0
$$

Equation (49) then gives a formulation for the ratio $c_{r}=c_{\max } / c_{\min }$,

$$
c_{r}=\frac{c_{\max }}{c_{\min }}=\frac{\gamma^{-} e^{\gamma^{-} h}-\gamma^{+} e^{\gamma^{+} h}}{\left(\gamma^{-}-\gamma^{+}\right) e^{\left(\gamma^{+}+\gamma^{-}\right) h}} .
$$

Example values are $c_{\min }=5 \times 10^{-2} \mathrm{~mol} \mathrm{~m}{ }^{-3}$ and $c_{\max }=0.1 \mathrm{~mol} \mathrm{~m}^{-3}$, giving $c_{r}=2$, although any desired value of $c_{r}$ could be investigated using (51). The result (51) shows that it is the prescription of the ratio $c_{r}$ that is crucial in determining the properties of the construct system, rather than particular choices for $c_{\min }$ or $c_{\max }$. It is therefore relevant to test how the ratio $c_{r}$ varies as a function of the experimentally controlled parameters. In particular, the printed cell density $\phi$, and the pressure drop across the scaffold $\Delta P$ are chosen by experimentalists as part of the construct design, and (51) gives a formulation for computing viable values for $\phi$ and $\Delta P$ given a prescribed value of $c_{r}$.

The pressure drop $\Delta P$ is re-dimensionalised in the following results using the typical viscous pressure scaling $P=\mu U a / r^{2}$ where $r=2 d, \mu, d$ and $a$ are given in Table 1 , and the velocity scale $U$ is chosen to be $U_{\min }=1.65 \times 10^{-7} \mathrm{~m} \mathrm{~s}^{-1}$ as identified in Section3.

The ratio $c_{r}$ varies as a function of the cell density $\phi$ through the dependence of $\gamma^{ \pm}$on the Dämkohler number, $\mathrm{Da}_{G}^{c}=k_{c} a^{2} \phi / D_{m}$. Figure 6 shows a plot of the variation in the ratio $c_{r}$ as a function of the printed cell density $\phi$ when $k_{c}=1.2 \times 10^{-17} \mathrm{~m}^{3} \mathrm{~s}^{-1}$ cell ${ }^{-1}, a=5 \times 10^{-3} \mathrm{~m}, D_{m}=3 \times 10^{-9} \mathrm{~m}^{2} \mathrm{~s}^{-1}$ and the (dimensional) pressure drop is fixed at $\Delta P=1.4 \times 10^{-3} \mathrm{~N} \mathrm{~m}^{-2}$. As the cell density $\phi$ increases so does the ratio $c_{r}$; this is the result of an increased demand of oxygen consumption that requires more oxygen to be supplied at the inlet relative to the outlet.

Similarly, the ratio $c_{r}$ also varies as a function of the applied pressure drop $\Delta P$ through the dependence of $\gamma^{ \pm}$on the velocity component $\langle w\rangle$. Figure 7 shows a plot of the variation in the ratio $c_{r}$ as a function of $\Delta P$ when the printed cell density is fixed at an example value $\phi=6 \times 10^{14} \mathrm{cells} \mathrm{m}^{-3}$. The ratio $c_{r}$ decreases as the pressure drop increases as this induces a higher flux of medium through the construct, and so the depletion of nutrients is reduced.

Further, for a prescribed value of $c_{r}$ (51) now gives a direct relationship between $\Delta P$ and $\phi$ and so by fixing $\Delta P$ based on the analysis of Sections 3-4, the precise value of $\phi$ can be determined (rather than just the maximum value determined from condition (35)). For example, the analysis of Sections 3-4 may impose a pressure drop of $\Delta P=1.4 \times 10^{-3} \mathrm{~N} \mathrm{~m}^{-3}$ across the construct. Equation (51) can then

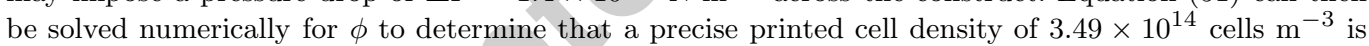
required to maintain an inlet-to-outlet concentration ratio of $c_{r}=2$. This process can be repeated for any desired value of $c_{r}$.

\section{Conclusions}

The analysis presented in Sections 3-5 has provided a number of criteria which may be used to guide the design of the tissue engineering construct. In this Section we will summarise the various results and provide recommendations for optimising the scaffold properties.

If we assume that the macroscopic dimensions of the construct - namely the length $a$ of strands and the depth $h$ - are prescribed, the remaining design parameters to be decided are:

1. The cell density $\phi$;

2. The scaffold geometry, including the configuration (as shown in Figure 1), and the strand diameter $d$ and separation $r$;

3. The mean flow velocity $\langle w\rangle$ and initial concentration of oxygen, $c_{0}$. 


\section{ACCEPTED MANUSCRIPT}

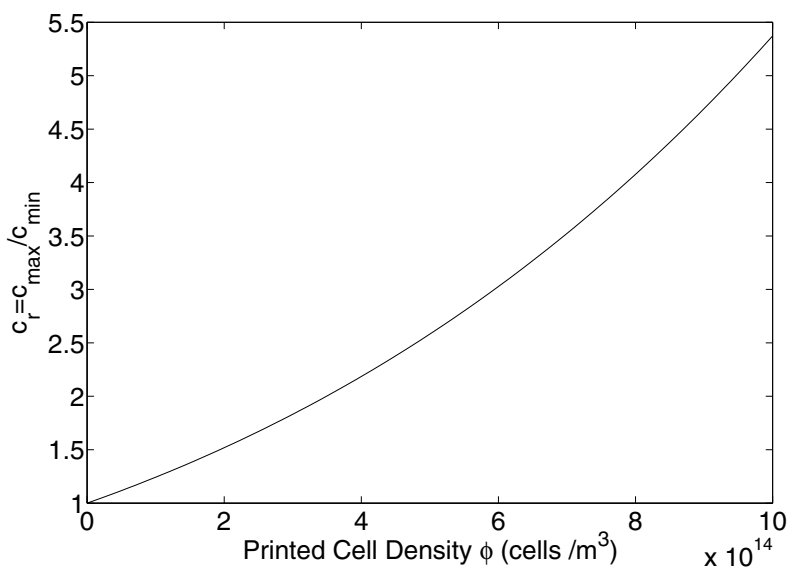

Fig. 6 The ratio $c_{r}=c_{\max } / c_{\min }$, as a function of the cell density $\phi$, when $\mathrm{Pe}_{G}^{c}=0.625, \bar{D}=0.87$, and $\Delta P=1.4 \times 10^{-3} \mathrm{~N} \mathrm{~m}^{-3}$.

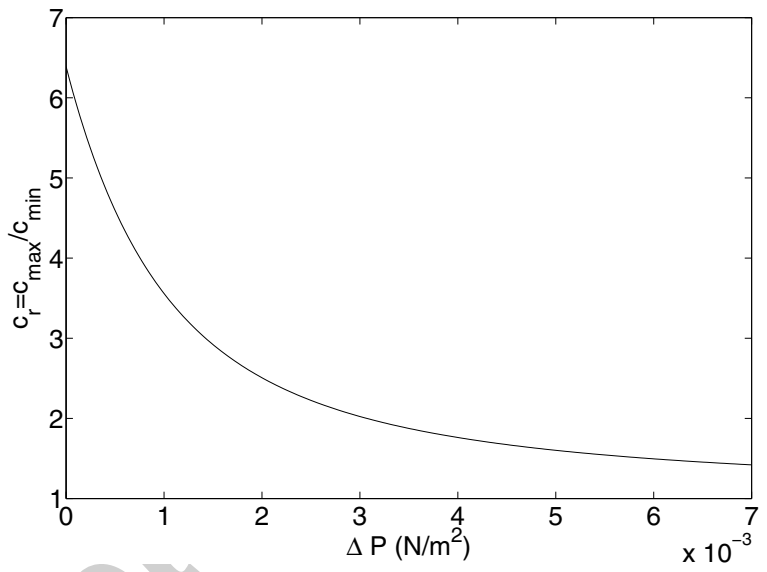

Fig. 7 The ratio $c_{r}=c_{\max } / c_{\min }$, as a function of the pressure drop across the construct $\Delta P$, when $\operatorname{Pe}_{G}^{c}=0.625$, $\bar{D}=0.87$, and $\phi=6 \times 10^{14}$ cells $\mathrm{m}^{-3}$.

The results from the homogenization analysis for the oxygen, glucose and lactate transport indicate that for given values of $k_{c}, k_{g}$ and $k_{l}$, there is a maximum bound on the value of $\phi$ for sufficient oxygen and glucose supply, and for lactate removal. Specifically, if the local Damköhler number in these expressions must be at least $\mathcal{O}\left(\epsilon^{2}\right)$, we obtain (35), which relates the maximum value of $\phi$ to the diffusion, consumption and production parameters, and accordingly allows for an optimal choice of cell density.

Regarding the scaffold geometry, the results of Section 4 indicate that the most favourable configuration of strands is the waffle structure (Figure 1c) and that perfusion in the vertical direction is preferable. These conclusions were drawn based on homogenization results shown in Figures 3-4.

The diameter of the strands forming the gel scaffold is limited by the requirement that glucose and oxygen are able to diffuse throughout the material. Concurrently, lactate must be able to diffuse out of the gel so that the cells are not poisoned. These considerations gave rise to the condition (14), which describes the maximum value of the strands' diameter, $d_{\max }$. In comparison, the lower bound for $d$ is 
only limited by the capabilities of the printing device that creates the strands. Thus a wide range of strand diameters may be permitted by our analysis. However, we must note that the later multiple-scales calculations depended on $r / a=\epsilon \ll 1$, and so assuming that $d \sim r$, it is also necessary that $d_{\max } \ll a$ for the analysis in this paper to hold.

Next, we determine the optimum values for the perfusion velocity through the construct and the separation of strands. These may be determined simultaneously as follows. The minimum velocity is based on the need to advect lactate out of the construct. As can be seen from Figure 5c, the lactate concentration builds up through the construct to a maximum at the outlet. The velocity needs to be high enough that the outlet concentration does not reach toxic levels. Condition (19) is a simple expression of this requirement; a more accurate representative value may be obtained using expression (47), determining the velocity for which $\langle l\rangle$ remains at a non-toxic level. The latter expression will provide a lower bound for the velocity $\langle w\rangle$ as a function of the solid fraction $n_{s}$, which (given $d$ ) is solely dependent on $r$. In [3], the authors use mechanical considerations to calculate that the maximum medium velocity that can be tolerated by the scaffold is $\mathcal{O}(1) \mathrm{ms}^{-1}$. One may therefore obtain the optimal perfusion velocity and solid fraction by choosing the maximum value of $n_{s}$ for which $\langle w\rangle_{\min }$ is smaller than this. Through (27), which describes the dependence of $n_{s}$ on the strand diameter, $d$, and separation, $r$, we then find the optimal strand separation.

Finally it is necessary to maintain both a minimum and maximum value for the oxygen concentration in the construct, so that neither ECM production nor chondrogenic differentiation are hindered. Based on this, the ratio of the inlet-to-outlet oxygen concentrations, $c_{r}$, is known and given by equation (51). This provides a method of evaluating the exact printed cell density $\phi$ required for a given value of $\Delta P$ determined through the perfusion analysis.

We have therefore determined a strategy for designing a tissue engineering construct, using the powerful mathematical technique of homogenization. The bounds on the controllable elements of design that have been derived here should be observed to optimise the growth and function of the cartilage tissue being manufactured. This reduces the "trial and error" aspect of construct design and thus contributes to the long-term aim of tailoring cartilage tissue to an individual patient's needs.

Acknowledgements This paper was conceived as an extension to a problem considered at the 7th Mathematics in Medicine Study Group [3], held at the University of Southampton in September 2007, with funding from the Engineering and Physical Sciences Research Council (EPSRC). We would like to thank the participants who worked on different aspects of the problem, in particular Ovidiu Bagdasar, Louise Dyson, Dr Liesbet Geris, Prof. Kerry Landman, Jonathan Moles, Sylvian Reboux, Dr Zimei Rong, Dr Tiina Roose and Muhammad Shakeel.

Dr. Malda is supported by a VENI fellowship from the Dutch Technology Foundation STW, Applied Science Division of NWO and the Technology Program of the Ministry of Economic Affairs.

We would like to thank Dr Sarah Waters for many helpful discussions, and in addition the reviewer for their comments and suggestions.

\section{Appendix A: Medium Flow Homogenization}

Here we detail the asymptotic homogenization process that determines the effective transport equations for the culture medium, given by (22)-(23). This analysis is based on the scaffold property that the strand separation $r$ (measured between strand centres) is much smaller than the construct dimensions, so that

$$
\epsilon=\frac{r}{a} \ll 1,
$$

Based on this, the two length scales characterized by $r$ (the micro- or local scale) and $a$ (the macro- or global scale) are well-separated. Assuming that the micro-scale structure is periodic (as is certainly valid for the scaffold structures displayed in Figure 1) and that the medium flow properties on the microscale are known, it is then possible to use asymptotic techniques to move from the local to the global 
descriptions and derive the effective flow equations on the macro-scale. The analysis that follows for the culture medium flow is not novel (see, for example, $[4,13]$ ), however it has not been applied to tissue engineering previously and is detailed here for the ease of a new audience.

We consider a periodic cell with dimension $r$ and denote its total volume by $V$, comprised of the culture medium volume $V_{m}$ and scaffold volume $V_{s}\left(V=V_{m} \cup V_{s}\right)$. The interface between $V_{m}$ and $V_{s}$ is denoted by $\Sigma$. The culture medium is a Newtonian fluid described by the Navier-Stokes equations (1)-(2) in $V_{m}$ subject to the no-slip boundary condition (3) on the interface $\Sigma$. We non-dimensionalise equations (1)-(3) on the local lengthscale, with the viscous rescaling

$$
\mathbf{x}=r \mathbf{x}^{\prime}, \mathbf{u}=U \mathbf{u}^{\prime}, p=\frac{\mu U a}{r^{2}} p^{\prime}+p_{0}, t=\frac{a}{U} t^{\prime},
$$

and define the Reynolds number $\operatorname{Re}=\rho U r / \mu$ as the dimensionless parameter representing the ratio of inertial to viscous forces in the medium. The typical velocity scale $U$ is not known experimentally, although the previous calculation (20) suggests that $U=\mathcal{O}\left(10^{-7}\right) \mathrm{m} \mathrm{s}^{-1}$ is a minimum requirement to ensure lactate removal from the construct. We seek the system behaviour on the global lengthscale, and so in anticipation of the nutrient/waste product transport problem the timescale chosen corresponds to global advection. Two considerations motivate the pressure scale; firstly the Reynolds number is small so a scaling that balances pressure and viscous effects is appropriate. Secondly, in the homogenization limit $\epsilon \rightarrow 0$ the medium flow region becomes constricted, so a corresponding decrease in viscosity is required to maintain flow rates; indeed the ratio of permeability to viscosity should be fixed so viscosity should be rescaled with $\epsilon^{-2}$. The appropriate pressure scale is therefore $p=\epsilon^{-2} \mu U / r+p_{0}$, where $p_{0}$ is the ambient pressure. Given the parameter values in Table 1, and assuming the strand separation $r=2 d$,

$$
\begin{aligned}
\epsilon & =0.08, \\
\operatorname{Re} & =4 \times 10^{-5}=\mathcal{O}\left(\epsilon^{4}\right) .
\end{aligned}
$$

The Reynolds number Re is very small, indicating that viscous forces dominate over inertial forces in the fluid. Dropping the primes, the system becomes

$$
\begin{aligned}
\boldsymbol{\nabla} \cdot \mathbf{u} & =0 \text { in } V_{m}, \\
\epsilon \operatorname{Re}\left(\epsilon \frac{\partial \mathbf{u}}{\partial t}+(\mathbf{u} \cdot \boldsymbol{\nabla}) \mathbf{u}\right) & =-\boldsymbol{\nabla} p+\epsilon \nabla^{2} \mathbf{u} \text { in } V_{m}, \\
\mathbf{u} & =\mathbf{0} \text { on } \Sigma .
\end{aligned}
$$

As $\epsilon \ll 1$, the local and global length scales are well-separated. We denote the separate micro- and macroposition vectors by $\mathbf{x}$ and $\mathbf{X}$ respectively, and relate them through $\mathbf{X}=\epsilon \mathbf{x}$. Under the assumption of scale separation $\mathbf{x}$ and $\mathbf{X}$ may be treated as independent variables and so we expand the gradient and Laplacian operators through

$$
\boldsymbol{\nabla}=\boldsymbol{\nabla}_{x}+\epsilon \boldsymbol{\nabla}_{X}, \nabla^{2}=\nabla_{x}^{2}+2 \epsilon \boldsymbol{\nabla}_{x} \cdot \nabla_{X}+\epsilon^{2} \nabla_{X}^{2}
$$

Justifying the validity of this assumption in the limit as $\epsilon \rightarrow 0$ is one of the main issues in rigorous homogenization theory $[5,38]$. We now denote the medium-scaffold boundary by $\Sigma_{\epsilon}$, as it varies on the local length scale. The rescaled equations in $V_{m}$ become

$$
\begin{aligned}
\boldsymbol{\nabla}_{x} \cdot \mathbf{u}+\epsilon \boldsymbol{\nabla}_{X} \cdot \mathbf{u}= & 0 \\
\epsilon \operatorname{Re}\left(\epsilon \frac{\partial \mathbf{u}}{\partial t}+\left(\mathbf{u} \cdot \boldsymbol{\nabla}_{x}\right) \mathbf{u}+\epsilon\left(\mathbf{u} \cdot \boldsymbol{\nabla}_{X}\right) \mathbf{u}\right)= & -\boldsymbol{\nabla}_{x} p-\epsilon \boldsymbol{\nabla}_{X} p+\epsilon \nabla_{x}^{2} \mathbf{u} \\
& +2 \epsilon^{2} \boldsymbol{\nabla}_{X} \cdot \boldsymbol{\nabla}_{x} \mathbf{u}+\epsilon^{3} \nabla_{X}^{2} \mathbf{u}
\end{aligned}
$$

The boundary condition remains $\mathbf{u}=\mathbf{0}$ on $\Sigma_{\epsilon}$. We perform multiple scales expansions

$$
\mathbf{u}=\mathbf{u}^{(0)}(\mathbf{x}, \mathbf{X})+\epsilon \mathbf{u}^{(1)}(\mathbf{x}, \mathbf{X})+\ldots, p=p^{(0)}(\mathbf{x}, \mathbf{X})+\epsilon p^{(1)}(\mathbf{x}, \mathbf{X})+\ldots
$$


where the $\mathbf{u}^{(i)}$ and $p^{(i)}(i=0,1, \ldots)$ are assumed to be periodic in $\mathbf{x}$. Equating powers of $\epsilon$ (to avoid singularity in the limit as $\epsilon \rightarrow 0$ ) in equation (60), we find

$$
\begin{aligned}
\boldsymbol{\nabla}_{x} \cdot \mathbf{u}^{(0)} & =0, \\
\boldsymbol{\nabla}_{x} \cdot \mathbf{u}^{(1)}+\boldsymbol{\nabla}_{X} \cdot \mathbf{u}^{(0)} & =0
\end{aligned}
$$

and, in equation (61), we find

$$
\begin{aligned}
\boldsymbol{\nabla}_{x} p^{(0)} & =0 \Rightarrow p^{(0)}=p^{(0)}(\mathbf{X}), \\
\nabla_{x} p^{(1)}-\nabla_{x}^{2} \mathbf{u}^{(0)} & =-\nabla_{X} p^{(0)} .
\end{aligned}
$$

Finally the boundary condition gives

$$
\mathbf{u}^{(m)}=0 \text { on } \Sigma_{\epsilon}, \text { for } m=0,1, \ldots .
$$

Firstly note that, from $(65), p^{(0)}$ is a function of the global variable $\mathbf{X}$ only and so is constant on the local scale. Hence, $p^{(1)}$ can be thought of as the local adjustment to $p^{(0)}$. Equations (63)-(67) define a sequence of boundary-value problems in the periodic cell. We firstly solve for $\mathbf{u}^{(0)}$ and $p^{(1)}$ using equations (63), (66) and (67). Motivated by the independence of the micro- and macro- variables $\mathbf{x}$ and $\mathbf{X}$, we separate $\mathbf{u}^{(0)}$ and $p^{(1)}$ into components that are either constant or varying locally, and evaluate the local variation by solving various boundary value problems on the periodic cell. Integrating the resulting expressions for $\mathbf{u}^{(0)}$ and $p^{(1)}$ over the periodic domain provides averaged transport equations that describe the velocity and pressure variations on the macro-scale; the dependence on the micro-scale configuration occurs through the reliance of the transport coefficients on the locally varying components of $\mathbf{u}^{(0)}$ and $p^{(1)}$. The averaged transport equations must finally be solved on the global domain so that the dependence of the problem on both length scales has been evaluated.

In the case here, this process is facilitated by the fact that the equations (63), (66) and (67) are linear, and the forcing term $-\boldsymbol{\nabla}_{X} p^{(0)}$ in equation (66) is dependent on $\mathbf{X}$ only. This motivates solutions of the form

$$
\mathbf{u}^{(0)}=-\frac{\partial p^{(0)}}{\partial X_{j}} \mathbf{w}^{j}, p^{(1)}=-P_{j} \frac{\partial p^{(0)}}{\partial X_{j}}+\bar{p}^{(1)},
$$

where $\mathbf{w}^{j}(\mathbf{x}), P_{j}(\mathbf{x})$ are unknown functions of the local length variable and account for the local variations in $\mathbf{u}^{(0)}$ and $p^{(1)}$. The index $j=1,2,3$ identifies the Cartesian components of $\mathbf{x}$ (corresponding to unit vectors $\mathbf{e}_{j}$ in the $j$-th direction) and the summation convention is assumed. The term $\bar{p}^{(1)}(\mathbf{X})$ is constant locally and is included here for completeness, but does not need to be determined unless the $\mathcal{O}(\epsilon)$ correction $\mathbf{u}^{(1)}$ to the velocity profile is required. The variables $\mathbf{w}^{j}$ and $P_{j}$ are then determined by substituting (68) into (63), (66), (67) to give the cell problem

$$
\begin{aligned}
\boldsymbol{\nabla}_{x} \cdot \mathbf{w}^{j} & =0 \text { in } V_{m}, \\
\boldsymbol{\nabla}_{x} P_{j} & =\nabla_{x}^{2} \mathbf{w}^{j}+\mathbf{e}_{j} \text { in } V_{m}, \\
\mathbf{w}^{j} & =0 \text { on } \Sigma_{\epsilon}(\mathbf{x})=0,
\end{aligned}
$$

with $\mathbf{w}^{j}$ and $P_{j}$ periodic in $\mathbf{x}$. This canonical cell problem (69)-(71) could be solved numerically for a prescribed micro-scale configuration, as in Section 4. Once solved, we take averages over the fluid medium domain with respect to $\mathbf{x}$, defined by

$$
\langle g\rangle=\frac{1}{|V|} \iiint_{V_{m}} g \mathrm{~d} V
$$

where $|V|$ is the total volume of the periodic cell. Defining

$$
\kappa_{i j}=\frac{1}{|V|} \iiint_{V_{m}} w_{i}^{j} \mathrm{~d} V
$$


we then take averages of the leading-order velocity expression (68) to give Darcy's Law,

$$
\left\langle\mathbf{u}^{(0)}\right\rangle=-\kappa \nabla_{X} p^{(0)} .
$$

Therefore $\boldsymbol{\kappa}$ is the (constant) permeability tensor associated with the construct, which behaves as a porous medium. Finally, taking averages of the $\mathcal{O}(\epsilon)$ divergence equation given in (64) yields

$$
\iiint_{V_{m}} \boldsymbol{\nabla}_{x} \cdot \mathbf{u}^{(1)} \mathrm{d} V=-\boldsymbol{\nabla}_{X} \cdot\left(\iiint_{V_{m}} \mathbf{u}^{(0)} \mathrm{d} V\right) .
$$

Using the divergence theorem, boundary condition (67) with $m=1$ and the periodicity of $\mathbf{u}^{(1)}$ on the cell,

$$
\iiint_{V_{m}} \boldsymbol{\nabla}_{x} \cdot \mathbf{u}^{(1)} \mathrm{d} V=\iint_{\Sigma_{\epsilon}} \mathbf{u}^{(1)} \cdot \mathbf{n} \mathrm{d} S=0 .
$$

Hence,

$$
\nabla_{X} \cdot\left\langle\mathbf{u}^{(0)}\right\rangle=0
$$

Equations (74) and (77) combine to give a single equation for the evolution of the fluid pressure on the macro-scale,

$$
\boldsymbol{\nabla}_{X} \cdot\left(\boldsymbol{\kappa} \boldsymbol{\nabla}_{X} p^{(0)}\right)=0
$$

This is solved for $p^{(0)}$ in Section 4 for the specific micro-scale geometries identified by Figure 1, and the associated fluid velocities in the construct are then also fully determined.

\section{Appendix B: Homogenization of Oxygen, Glucose and Lactate Transport}

We perform the homogenization process for oxygen transport in detail here, and then state the corresponding results for glucose and lactate as natural extensions.

The dimensional equations describing the oxygen transport in the periodic cell are written in conservation form as

$$
\begin{aligned}
\frac{\partial c_{m}}{\partial t}+\boldsymbol{\nabla} \cdot\left(c_{m} \mathbf{u}-D_{m} \boldsymbol{\nabla} c_{m}\right) & =0 \text { in } V_{m}, \\
\frac{\partial c_{s}}{\partial t}-\boldsymbol{\nabla} \cdot\left(D_{s} \boldsymbol{\nabla} c_{s}\right) & =-k_{c} \phi c_{s} \text { in } V_{s}, \\
c_{m} & =c_{s} \text { on } \Sigma, \\
D_{m} \boldsymbol{\nabla} c_{m} \cdot \mathbf{n} & =D_{s} \boldsymbol{\nabla} c_{s} \cdot \mathbf{n} \text { on } \Sigma .
\end{aligned}
$$

We non-dimensionalise these using the scalings (53) and $c=c_{0} c^{\prime}, D=D_{m} D^{\prime}$, where $c_{0}$ is a typical inlet concentration, and define dimensionless parameters

$$
\bar{D}=\frac{D_{s}}{D_{m}}, \operatorname{Pe}_{L}^{c}=\frac{U r}{D_{m}}, \mathrm{Da}_{L}^{c}=\frac{k_{c} r^{2} \phi}{D_{m}} .
$$

The dimensionless number $\bar{D}$ represents the ratio of diffusion coefficients. The local Péclet number for oxygen, $\mathrm{Pe}_{L}^{c}$, represents the ratio of diffusive to advective timescales on the local lengthscale $d$. The local Damköhler number for oxygen, $\mathrm{Da}_{L}^{c}$, represents the ratio of diffusive to consumptive timescales, again on the local lengthscale $d$. Using the parameter values in Table 1 we estimate $\bar{D}=0.87=\mathcal{O}(1)$. Using the velocity scale $U=1.65 \times 10^{-7} \mathrm{~m} \mathrm{~s}^{-1}$ from (20), we estimate $\mathrm{Pe}_{L}^{c}=0.02=\mathcal{O}(\epsilon)$, so diffusion dominates over advection on the local lengthscale. The global Péclet number is defined as the ratio of diffusive to advective timescales on the global lengthscale and satisfies

$$
\mathrm{Pe}_{G}^{c}=\frac{U a}{D_{m}} \Rightarrow \mathrm{Pe}_{L}^{c}=\epsilon \mathrm{Pe}_{G}^{c}
$$


The global Péclet number, $\mathrm{Pe}_{G}^{c}$, is therefore $\mathcal{O}(1)$ as expected from Table 3 (advection and diffusion balance each other on the global scale). In contrast, the size of the local Damköhler number $\mathrm{Da}_{L}^{c}$ depends on the consumption rate $k_{c}$ and cell density $\phi$ in the gel. The global Damköhler number $\mathrm{Da}_{G}^{c}$ represents the ratio of diffusive to consumptive timescales on the global lengthscale $a$ and is given by

$$
\operatorname{Da}_{G}^{c}=\frac{k_{c} a^{2} \phi}{D_{m}}
$$

As discussed in Section 5, $\mathrm{Da}_{L}^{c}=\epsilon^{2} \mathrm{Da}_{G}^{c}$ is at largest $\mathcal{O}\left(\epsilon^{2}\right)$ in order for sufficient oxygen to be supplied to the cells to meet the demands of consumption. Table 4 shows how the order of magnitude of $\mathrm{Da}_{L}^{c}$ varies with the product $k_{c} \phi$, and $\mathrm{Da}_{L}^{c}=\mathcal{O}\left(\epsilon^{2}\right)$ or smaller is typical. Using the fact that $\boldsymbol{\nabla} \cdot \mathbf{u}=0$ and dropping the primes, the non-dimensionalised system is given by

$$
\begin{aligned}
\epsilon \operatorname{Pe}_{G}^{c}\left(\epsilon \frac{\partial c_{m}}{\partial t}+\mathbf{u} \cdot \nabla c_{m}\right) & =\nabla^{2} c_{m} \text { in } V_{m} \\
\epsilon^{2} \operatorname{Pe}_{G}^{c} \frac{\partial c_{s}}{\partial t} & =\bar{D} \nabla^{2} c_{s}-\epsilon^{2} \operatorname{Da}_{G}^{c} c_{s} \text { in } V_{s}, \\
c_{m} & =c_{s} \text { on } \Sigma \\
\nabla c_{m} \cdot \mathbf{n} & =\bar{D} \nabla c_{s} \cdot \mathbf{n} \text { on } \Sigma
\end{aligned}
$$

The multiple scales technique is now used and independent local and global position vectors $\mathbf{x}$ and $\mathbf{X}$ (related through $\mathbf{X}=\epsilon \mathbf{x}$ ) are introduced. Expanding the gradient and Laplacian operators through (59) gives

$$
\begin{aligned}
\epsilon \operatorname{Pe}_{G}^{c}\left(\epsilon \frac{\partial c_{m}}{\partial t}+\mathbf{u} \cdot \boldsymbol{\nabla}_{x} c_{m}+\epsilon \mathbf{u} \cdot \boldsymbol{\nabla}_{X} c_{m}\right)= & \nabla_{x}^{2} c_{m}+2 \epsilon \boldsymbol{\nabla}_{X} \cdot \boldsymbol{\nabla}_{x} c_{m}+\epsilon^{2} \nabla_{X}^{2} c_{m} \text { in } V_{m}, \\
\epsilon^{2} \operatorname{Pe}_{G}^{c} \frac{\partial c_{s}}{\partial t}= & \bar{D} \nabla_{x}^{2} c_{s}+2 \epsilon \bar{D} \nabla_{X} \cdot \nabla_{x} c_{s}+\epsilon^{2} \bar{D} \nabla_{X}^{2} c_{s} \\
& -\epsilon^{2} \operatorname{Da}_{G}^{c} c_{s} \text { in } V_{s} \\
c_{m} & =c_{s} \text { on } \Sigma_{\epsilon} \\
\boldsymbol{\nabla}_{x} c_{m} \cdot \mathbf{n}+\epsilon \boldsymbol{\nabla}_{X} c_{m} \cdot \mathbf{n} & =\bar{D} \boldsymbol{\nabla}_{x} c_{s} \cdot \mathbf{n}+\epsilon \bar{D} \boldsymbol{\nabla}_{X} c_{s} \cdot \mathbf{n} \text { on } \Sigma_{\epsilon} .
\end{aligned}
$$

We expand $\mathbf{u}$ in powers of $\epsilon$ as in (62) and the oxygen concentration $c$ as

$$
c=c^{(0)}(\mathbf{x}, \mathbf{X})+\epsilon c^{(1)}(\mathbf{x}, \mathbf{X})+\ldots
$$

where the $c^{(i)}(i=0,1, \ldots)$ are assumed to be periodic in $\mathbf{x}$, and equate powers of $\epsilon$. The leading-order homogenized equation for the average concentration will include the time-derivative term, and this will occur at $\mathcal{O}\left(\epsilon^{2}\right)$ from equations (90) and (91). Therefore we expect that equating powers of $\epsilon^{0}$ and $\epsilon^{1}$ will provide the $\mathcal{O}(1)$ and $\mathcal{O}(\epsilon)$ contributions to the average concentration; equating powers of $\epsilon^{2}$ will provide the homogenized equation for the leading-order average concentration.

\begin{tabular}{ll}
\hline$k_{c} \phi$ & $\mathrm{Da}_{L}^{c}$ \\
\hline $1.2 \times 10^{-4}$ & $\mathcal{O}\left(\epsilon^{2}\right)$ \\
$1 \times 10^{-5}$ & $\mathcal{O}\left(\epsilon^{3}\right)$ \\
\hline
\end{tabular}

Table 4 Table comparing $k_{c} \phi$ with the local Damköhler number for oxygen $\mathrm{Da}_{L}$. 
Equating powers of $\epsilon^{0}$ yields

$$
\begin{aligned}
\nabla_{x}^{2} c_{m}^{(0)} & =0 \text { in } V_{m}, \\
\bar{D} \nabla_{x}^{2} c_{s}^{(0)} & =0 \text { in } V_{s}, \\
c_{m}^{(0)} & =c_{s}^{(0)} \text { on } \Sigma_{\epsilon}, \\
\nabla_{x} c_{m}^{(0)} \cdot \mathbf{n} & =\bar{D} \nabla_{x} c_{s}^{(0)} \cdot \mathbf{n} \text { on } \Sigma_{\epsilon} .
\end{aligned}
$$

The only possible solution of this system is that both $c_{m}^{(0)}$ and $c_{s}^{(0)}$ are constant on the local scale, and so are given by $c_{m}^{(0)}=c_{s}^{(0)}=\bar{c}(\mathbf{X}, t)$ where $\bar{c}(\mathbf{X}, t)$ is to be determined from a homogenized equation. Equating powers of $\epsilon$ in (90)-(93) and noting that $\bar{c}$ is independent of the local variable $\mathbf{x}$ gives

$$
\begin{aligned}
\nabla_{x}^{2} c_{m}^{(1)} & =0 \text { in } V_{m} \\
\bar{D} \nabla_{x}^{2} c_{s}^{(1)} & =0 \text { in } V_{s} \\
c_{m}^{(1)} & =c_{s}^{(1)} \text { on } \Sigma_{\epsilon} \\
\nabla_{x} c_{m}^{(1)} \cdot \mathbf{n} & =\bar{D} \nabla_{x} c_{s}^{(1)} \cdot \mathbf{n}+(\bar{D}-1) \nabla_{X} \bar{c} \cdot \mathbf{n} \text { on } \Sigma_{\epsilon} .
\end{aligned}
$$

The solutions for $c_{m}^{(1)}$ and $c_{s}^{(1)}$ are not constant due to the non-homogeneous Neumann boundary condition (102). We exploit the linearity of the system described by (99)-(102) and propose solutions of the form

$$
\begin{aligned}
c_{m}^{(1)} & =\boldsymbol{\chi}_{m}^{c}(\mathbf{x}) \cdot \nabla_{X} \bar{c}+\bar{c}^{(1)}(\mathbf{X}, t), \\
c_{s}^{(1)} & =\boldsymbol{\chi}_{s}^{c}(\mathbf{x}) \cdot \nabla_{X} \bar{c}+\bar{c}^{(1)}(\mathbf{X}, t) .
\end{aligned}
$$

The variables $\chi_{m}$ and $\chi_{s}$ can then be determined from the cell problems

$$
\begin{aligned}
\nabla_{x}^{2} \boldsymbol{\chi}_{m}^{c} & =\mathbf{0} \text { in } V_{m}, \\
\nabla_{x}^{2} \boldsymbol{\chi}_{s}^{c} & =\mathbf{0} \text { in } V_{s}, \\
\boldsymbol{\chi}_{m}^{c} & =\boldsymbol{\chi}_{s}^{c} \text { on } \Sigma_{\epsilon}, \\
\left(\boldsymbol{\nabla}_{x} \boldsymbol{\chi}_{m}^{c}\right)^{T} \cdot \mathbf{n}-\bar{D}\left(\boldsymbol{\nabla}_{x} \boldsymbol{\chi}_{s}^{c}\right)^{T} \cdot \mathbf{n} & =(\bar{D}-1) \mathbf{n} \text { on } \Sigma_{\epsilon} .
\end{aligned}
$$

A further condition is required to determine $c_{m}^{(1)}$ and $c_{s}^{(1)}$ uniquely and this condition is chosen to ensure that $\bar{c}$ is the leading-order concentration with an $\mathcal{O}(\epsilon)$ correction that is a function of the global variable only; this condition is given by

$$
\int_{V_{m}} \boldsymbol{\chi}_{m}^{c} \mathrm{~d} V+\int_{V_{s}} \boldsymbol{\chi}_{s}^{c} \mathrm{~d} V=0 .
$$

Next we seek a homogenized transport equation for $\bar{c}$ by considering the $\mathcal{O}\left(\epsilon^{2}\right)$ system given by

$$
\begin{aligned}
\nabla_{x}^{2} c_{m}^{(2)} & =\operatorname{Pe}_{G}^{c}\left(\frac{\partial \bar{c}}{\partial t}+\mathbf{u}^{(0)} \cdot \nabla_{x} c_{m}^{(1)}+\mathbf{u}^{(0)} \cdot \nabla_{X} \bar{c}\right)-2 \nabla_{X} \cdot \nabla_{x} c_{m}^{(1)}-\nabla_{X}^{2} \bar{c} \text { in } V_{m} \\
\bar{D} \nabla_{x}^{2} c_{s}^{(2)} & =\operatorname{Pe}_{G}^{c} \frac{\partial \bar{c}}{\partial t}-2 \bar{D} \nabla_{X} \cdot \nabla_{x} c_{s}^{(1)}-\bar{D} \nabla_{X}^{2} \bar{c}+\operatorname{Da}_{G}^{c} \bar{c} \text { in } V_{s} \\
c_{m}^{(2)} & =c_{s}^{(2)} \text { on } \Sigma_{\epsilon} \\
\boldsymbol{\nabla}_{x} c_{m}^{(2)} \cdot \mathbf{n}-\bar{D} \nabla_{x} c_{s}^{(2)} \cdot \mathbf{n} & =-\nabla_{X} c_{m}^{(1)} \cdot \mathbf{n}+\bar{D} \nabla_{X} c_{s}^{(1)} \cdot \mathbf{n} \text { on } \Sigma_{\epsilon}
\end{aligned}
$$

By the Fredholm Alternative, there is a solvability condition associated with this system which, if satisfied, ensures that a solution exists; this solvability condition gives the homogenized transport equation 
for $\bar{c}$. However, the invariant distribution associated with this system is locally constant, so the solvability condition reduces to integrating the system (110)-(111) over $V$. This process is discussed in further detail in [44]. For ease of notation, we define the variable $g$ through

$$
g(\mathbf{x}, \mathbf{X})=\left\{\begin{array}{cc}
g_{m}=\mathrm{Pe}_{G}^{c}\left(\frac{\partial \bar{c}}{\partial t}+\mathbf{u}^{(0)} \cdot \nabla_{x} c_{m}^{(1)}+\mathbf{u}^{(0)} \cdot \nabla_{X} \bar{c}\right)-2 \boldsymbol{\nabla}_{X} \cdot \boldsymbol{\nabla}_{x} c_{m}^{(1)}-\nabla_{X}^{2} \bar{c} \text { in } V_{m} \\
g_{s}=\operatorname{Pe}_{G}^{c} \frac{\partial \bar{c}}{\partial t}-2 \bar{D} \boldsymbol{\nabla}_{X} \cdot \boldsymbol{\nabla}_{x} c_{s}^{(1)}-\bar{D} \nabla_{X}^{2} \bar{c}+\mathrm{Da}_{G}^{c} \bar{c} & \text { in } V_{s}
\end{array} .\right.
$$

Using the flux boundary condition (113) the solvability condition becomes

$$
\int_{V_{s}} g_{s} \mathrm{~d} V+\int_{V_{m}} g_{m} \mathrm{~d} V=\int_{\Sigma_{\epsilon}}\left(\bar{D} \nabla_{X} c_{s}^{(1)}-\nabla_{X} c_{m}^{(1)}\right) \cdot \mathbf{n} \mathrm{d} S
$$

which can be simplified further to

$$
\int_{V_{s}} g_{s}+\nabla_{x} \cdot\left(\bar{D} \nabla_{X} c_{s}^{(1)}\right) \mathrm{d} V+\int_{V_{m}} g_{m}+\nabla_{x} \cdot \nabla_{X} c_{m}^{(1)} \mathrm{d} V=0
$$

Substituting in for $g_{s}$ and $g_{m}$ from (114) and dividing by $|V|$ yields

$$
\begin{aligned}
& \operatorname{Pe}_{G} \frac{\partial \bar{c}}{\partial t}+\operatorname{Pe}_{G}^{c}\left\langle\mathbf{u}^{(0)}\right\rangle \cdot \nabla_{X} \bar{c}+\frac{1}{|V|} \int_{V_{m}}\left(\operatorname{Pe}_{G}^{c} \mathbf{u}^{(0)} \cdot \nabla_{x} c_{m}^{(1)}-\nabla_{X} \cdot \nabla_{x} c_{m}^{(1)}\right) \mathrm{d} V \\
& -\frac{1}{|V|} \int_{V_{s}} \bar{D} \boldsymbol{\nabla}_{X} \cdot \nabla_{x} c_{s}^{(1)} \mathrm{d} V=\left(1+n_{s}(\bar{D}-1)\right) \nabla_{X}^{2} \bar{c}-n_{s} \operatorname{Da}_{G}^{c} \bar{c} .
\end{aligned}
$$

The integral terms of (118) may be evaluated as follows. Firstly,

$$
\begin{aligned}
\frac{\operatorname{Pe}_{G}^{c}}{|V|} \int_{V_{m}} \mathbf{u}^{(0)} \cdot \nabla_{x} c_{m}^{(1)} \mathrm{d} V & =\frac{\operatorname{Pe}_{G}^{c}}{|V|} \int_{V_{m}} \mathbf{u}^{(0)} \cdot \nabla_{x}\left(\chi_{m}^{c} \cdot \nabla_{X} \bar{c}+\bar{c}^{(1)}\right) \mathrm{d} V \\
& =\frac{\operatorname{Pe}_{G}^{c}}{|V|} \int_{V_{m}} \mathbf{u}^{(0)} \cdot \boldsymbol{\nabla}_{x}\left(\boldsymbol{\chi}_{m}^{c} \cdot \boldsymbol{\nabla}_{X} \bar{c}\right) \mathrm{d} V \\
& =\frac{\operatorname{Pe}_{G}}{|V|}\left(\int_{V_{m}}\left(\boldsymbol{\nabla}_{x} \boldsymbol{\chi}_{m}^{c}\right)^{T} \cdot \mathbf{u}^{(0)} \mathrm{d} V\right) \cdot \boldsymbol{\nabla}_{X} \bar{c}
\end{aligned}
$$

where the superscript $T$ denotes the transpose. In addition, we note that for a matrix $\mathbf{A}=A_{i j}$ and vector $\mathbf{b}=b_{i}$,

$$
\mathbf{A} \cdot \mathbf{b}=A_{i j} b_{i}
$$

and then in particular we can write

$$
\left(\boldsymbol{\nabla}_{x} \boldsymbol{\chi}_{m}^{c}\right)^{T} \cdot \mathbf{u}^{(0)}=\frac{\partial \chi_{m, j}}{\partial x_{i}} u_{i}^{(0)}
$$

Further,

$$
\begin{aligned}
-\frac{1}{|V|} \int_{V_{m}} \boldsymbol{\nabla}_{x} \cdot \boldsymbol{\nabla}_{X} c_{m}^{(1)} \mathrm{d} V & =-\frac{1}{|V|} \int_{V_{m}} \boldsymbol{\nabla}_{X} \cdot \boldsymbol{\nabla}_{x}\left(\boldsymbol{\chi}_{m}^{c} \cdot \boldsymbol{\nabla}_{X} \bar{c}+\bar{c}^{(1)}\right) \mathrm{d} V \\
& =-\frac{1}{|V|} \int_{V_{m}} \boldsymbol{\nabla}_{X} \cdot \boldsymbol{\nabla}_{x}\left(\boldsymbol{\chi}_{m}^{c} \cdot \boldsymbol{\nabla}_{X} \bar{c}\right) \mathrm{d} V \\
& =-\frac{1}{|V|} \boldsymbol{\nabla}_{X} \cdot\left(\left(\int_{V_{m}}\left(\boldsymbol{\nabla}_{x} \boldsymbol{\chi}_{m}^{c}\right)^{T} \mathrm{~d} V\right) \boldsymbol{\nabla}_{X} \bar{c}\right)
\end{aligned}
$$

Finally, by the same process

$$
-\frac{1}{|V|} \int_{V_{s}} \bar{D} \boldsymbol{\nabla}_{x} \cdot \boldsymbol{\nabla}_{X} c_{s}^{(1)} \mathrm{d} V=-\frac{1}{|V|} \boldsymbol{\nabla}_{X} \cdot\left(\left(\int_{V_{s}} \bar{D}\left(\boldsymbol{\nabla}_{x} \boldsymbol{\chi}_{s}^{c}\right)^{T} \mathrm{~d} V\right) \boldsymbol{\nabla}_{X} \bar{c}\right) .
$$


Therefore, the solvability condition (118) can be written in the form of an advection-diffusion-reaction equation for the leading-order average oxygen concentration $\langle c\rangle=\bar{c}+\mathcal{O}(\epsilon)$ as

$$
\operatorname{Pe}_{G}^{c}\left(\frac{\partial\langle c\rangle}{\partial t}+\tilde{\mathbf{u}}_{c} \cdot \nabla_{X}\langle c\rangle\right)=\nabla_{X} \cdot\left(\underline{\underline{\boldsymbol{\Omega}}}^{c} \boldsymbol{\nabla}_{X}\langle c\rangle\right)-n_{s} \operatorname{Da}_{G}^{c}\langle c\rangle,
$$

where the effective advective and diffusion coefficients are given by

$$
\begin{aligned}
\tilde{\mathbf{u}}_{c} & =\left\langle\mathbf{u}^{(0)}\right\rangle+\frac{1}{|V|} \int_{V_{m}}\left(\boldsymbol{\nabla}_{x} \boldsymbol{\chi}_{m}^{c}\right)^{T} \cdot \mathbf{u}^{(0)} \mathrm{d} V, \\
\underline{\underline{\boldsymbol{\Omega}}}^{c} & =\left(1+n_{s}(\bar{D}-1)\right) \underline{\underline{\mathbf{I}}}+\frac{1}{|V|} \int_{V_{m}}\left(\boldsymbol{\nabla}_{x} \boldsymbol{\chi}_{m}^{c}\right)^{T} \mathrm{~d} V+\frac{1}{|V|} \int_{V_{s}} \bar{D}\left(\boldsymbol{\nabla}_{x} \boldsymbol{\chi}_{s}^{c}\right)^{T} \mathrm{~d} V .
\end{aligned}
$$

From (128), the average oxygen concentration $\langle c\rangle$ advects with velocity $\tilde{\mathbf{u}}_{c}$, diffuses with tensor coefficient $\underline{\underline{\boldsymbol{\Omega}}}^{c}$ and is consumed with coefficient $\operatorname{Da}_{G}^{c} n_{s}$. Equation (128) should be solved in the construct subject to an appropriate initial condition

$$
\langle c\rangle(\mathbf{X}, 0)=c_{i n}(\mathbf{X}) .
$$

The analysis for glucose and lactate proceeds in the same way to give

$$
\begin{gathered}
\mathrm{Pe}_{G}^{g}\left(\frac{\partial\langle g\rangle}{\partial t}+\tilde{\mathbf{u}}_{g} \cdot \boldsymbol{\nabla}_{X}\langle g\rangle\right)=\nabla_{X} \cdot\left(\underline{\underline{\boldsymbol{\Omega}}}_{g} \boldsymbol{\nabla}_{X}\langle g\rangle\right)-n_{s} \mathrm{Da}_{G}^{g}\langle g\rangle, \\
\operatorname{Pe}_{G}^{l}\left(\frac{\partial\langle l\rangle}{\partial t}+\tilde{\mathbf{u}}_{l} \cdot \boldsymbol{\nabla}_{X}\langle l\rangle\right)=\boldsymbol{\nabla}_{X} \cdot\left(\underline{\underline{\boldsymbol{\Omega}}}_{l} \boldsymbol{\nabla}_{X}\langle l\rangle\right)+n_{s} \mathrm{Da}_{G}^{l},
\end{gathered}
$$

where the definitions of the advection and diffusion coefficients are naturally extended from (129)-(130). These should similarly be solved in the construct subject to appropriate initial conditions of the form

$$
\langle g\rangle(\mathbf{X}, 0)=g_{i n}(\mathbf{X}),\langle l\rangle(\mathbf{X}, 0)=l_{\text {in }}(\mathbf{X}) .
$$

\section{References}

1. T. Adachi, Y. Osako, M. Tanaka, M. Hojo, and S.J. Hollister. Framework for optimal design of porous scaffold microstructure by computational simulation of bone regeneration. Biomaterials, 27(21):3964-3972, 2006.

2. R.V. Allhands, P.A. Torzilli, and F.A. Kallfelz. Measurement of diffusion of uncharged molecules in articular cartilage. Cornell Vet., 74(2):111-123, 1984.

3. O. Bagdasar, C. Bailey, R. Carter, C. Catt, L. Dyson, R. Dyson, L. Geris, G. Jones, K. Landman, J. Moles, C. Please, S. Reboux, T. Roose, Z. Rong, B. Sengers, and M. Shakeel. Regenerative medicine using organ printing technologies. In In Proc. Seventh Mathematics in Medicine Study Group, University of Southampton, 2007.

4. J. Bear. Dynamics of Fluids in Porous Media. Courier Dover Publications, 1988.

5. A. Bensoussan, J.-L. Lions, and G.C. Papanicolaou. Asymptotic Analysis for Periodic Structures. North Holland, 1978.

6. R.G.M. Breuls, B.G. Sengers, C.W.J. Oomens, C.V.C. Bouten, and F.P.T. Baaijens. Predicting local cell deformations in engineered tissue constructs: A multilevel finite element approach. J. Biomech. Eng. - T. ASME, 124(2):198-207, 2002.

7. D.P. Byrne, D. Lacroix, J.A. Planell, D.J. Kelly, and P.J. Prendergast. Simulation of tissue differentiation in a scaffold as a function of porosity, Young's modulus and dissolution rate: Application of mechanobiological models in tissue engineering. Biomaterials, 28(36):5544-5554, 2007.

8. R.L. Carrier, M. Papadaki, M. Rupnick, F.J. Schoen, N. Bursac, R. Langer, L.E. Freed, and G. VunjakNovakovic. Cardiac tissue engineering: Cell seeding, cultivation parameters, and tissue construct characterization. Biotechnol. Bioeng., 64(5):580-589, 1999.

9. C.A. Chung, C.W. Chen, C.P. Chen, and C.S. Tseng. Enhancement of cell growth in tissue-engineering constructs under direct perfusion: Modeling and simulation. Biotechnol. Bioeng., 97(6):1603-1616, 2007.

10. E. deSilva and M.P.H. Stumpf. Complex networks and simple models in biology. J. R. Soc. Interface, 2(5):419-430, 2005.

11. J.C.Y. Dunn, W.-Y. Chan, V. Cristini, J.S. Kim, J. Lowengrub, S. Singh, and B.M. Wu. Analysis of cell growth in three-dimensional scaffolds. Tissue Eng., 12(4):705-716, 2006. 
12. N.E. Fedorovich, J.R. De Wijn, A.J. Verbout, J. Alblas, and W.J.A. Dhert. Three-Dimensional Fiber Deposition of Cell-Laden, Viable, Patterned Constructs for Bone Tissue Printing. Tissue Engineering Part A, 14(1):127-133, 2008.

13. AC Fowler. Mathematical Models in the Applied Sciences. Cambridge University Press, 1997.

14. C.J. Galban and B.R. Locke. Effects of spatial variation of cells and nutrient and product concentrations coupled with product inhibition on cell growth in a polymer scaffold. Biotechnol. Bioeng., 64(6):633-643, 1999.

15. F. Galbusera, M. Cioffi, M.T. Raimondi, and R. Pietrabissa. Computational modeling of combined cell population dynamics and oxygen transport in engineered tissue subject to interstitial perfusion. Computer Methods in Biomechanics and Biomedical Engineering, 10(4):279-287, 2007.

16. J.C. Haselgrove, I.M. Shapiro, and S.F. Silverton. Computer modeling of the oxygen supply and demand of cells of the avian growth cartilage. Am. J. Physiol._Cell Ph., 265(2):C497-C506, 1993.

17. H.K. Heywood, D.L. Bader, and D.A. Lee. Rate of oxygen consumption by isolated articular chondrocytes is sensitive to medium glucose concentration. J Cell Physiol, 206(2):402-10, 2006.

18. H.K. Heywood, P.K. Sembi, D.A. Lee, and D.L. Bader. Cellular utilization determines viability and matrix distribution profiles in chondrocyte-seeded alginate constructs. Tissue Eng., 10(9-10):1467-1479, 2004.

19. S.J. Hollister. Porous scaffold design for tissue engineering. Nat. Mater., 4(7):518-524, 2005.

20. S. Holm, A. Maroudas, J.P.G. Urban, G. Selstam, and A. Nachemson. Nutrition of the intervertebral disc: Solute transport and metabolism. Connect. Tissue Res., 8(2):101-119, 1981.

21. D.J. Kelly and P.J. Prendergast. Effect of a degraded core on the mechanical behaviour of tissue engineered cartilage constructs: A poro-elastic finite element analysis. Med. Biol. Eng. Comput., 42(1):9-13, 2004.

22. M.C. Lewis, B.D. MacArthur, J. Malda, G. Pettet, and C.P. Please. Heterogeneous proliferation within engineered cartilaginous tissue: The role of oxygen tension. Biotechnol. Bioeng., 91(5):607-615, 2005.

23. E.N. Lightfoot and K.A. Duca. The role of mass transfer in tissue function. In J.D. Bronzino, editor, The Biomedical Engineering Handbook, chapter 115. CRC Press, London, second edition, 2000.

24. J. Malda, J. Rouwkema, D.E. Martens, E.P. le Comte, F.K. Kooy, J. Tramper, C.A. van Blitterswijk, and J. Riesle. Oxygen gradients in tissue-engineered PEGT/PBT cartilaginous constructs: Measurement and modeling. Biotechnol. Bioeng., 86(1):9-18, 2004.

25. J. Malda, T.B.F. Woodfield, F. van der Vloodt, F.K. Kooy, D.E. Martens, J. Tramper, C.A. van Blitterswijk, and J. Riesle. The effect of PEGT/PBT scaffold architecture on oxygen gradients in tissue engineered cartilaginous constructs. Biomaterials, 25(26):5773-5780, 2004.

26. J. Malda, T.B.F. Woodfield, F. van der Vloodt, C. Wilson, D.E. Martens, J. Tramper, C.A. van Blitterswijk, and J. Riesle. The effect of PEGT/PBT scaffold architecture on the composition of tissue engineered cartilage. Biomaterials, 26(1):63-72, 2005.

27. I. Martin, S. Miot, A. Barbero, M. Jakob, and D. Wendt. Osteochondral tissue engineering. J. Biomech., 40(4):750-765, 2007.

28. I. Martin, B. Obradovic, L.E. Freed, and G. Vunjak-Novakovic. Method for quantitative analysis of glycosaminoglycan distribution in cultured natural and engineered cartilage. Ann. Biomed. Eng., 27(5):656-662, 1999.

29. I. Martin, B. Obradovic, S. Treppo, A.J. Grodzinsky, R. Langer, L.E. Freed, and G. Vunjak-Novakovic. Modulation of the mechanical properties of tissue engineered cartilage. Biorheology, 37(1-2):141-147, 2000.

30. V. Mironov, T. Boland, T. Trusk, G. Forgacs, and R.R. Markwald. Organ printing: Computer-aided jet-based 3D tissue engineering. Trends Biotechnol., 21(4):157-161, 2003.

31. D. Mokhbi Soukane, A. Shirazi-Adl, and J.P.G. Urban. Computation of coupled diffusion of oxygen, glucose and lactic acid in an intervertebral disc. J. Biomech., 40(12):2645-2654, 2007.

32. B. Obradovic, R.L. Carrier, G. Vunjak-Novakovic, and L.E. Freed. Gas exchange is essential for bioreactor cultivation of tissue engineered cartilage. Biotechnol. Bioeng., 63(2):197-205, 1999.

33. B. Obradovic, J.H. Meldon, L.E. Freed, and G. Vunjak-Novakovic. Glycosaminoglycan deposition in engineered cartilage: Experiments and mathematical model. AIChE J., 46(9):1860-1871, 2000.

34. M. Pisu, N. Lai, A. Concas, and G. Cao. A novel simulation model for engineered cartilage growth in static systems. Tissue Eng., 12(8):2311-2320, 2006.

35. B. Porter, R. Zauel, H. Stockman, R. Guldberg, and D. Fyhrie. 3-D computational modeling of media flow through scaffolds in a perfusion bioreactor. J. Biomech., 38(3):543-549, 2005.

36. M. Radisic, J. Malda, E. Epping, W. Geng, R. Langer, and G. Vunjak-Novakovic. Oxygen gradients correlate with cell density and cell viability in engineered cardiac tissue. Biotechnol. Bioeng., 93(2):332-343, 2006.

37. M.T. Raimondi, F. Boschetti, L. Falcone, G.B. Fiore, A. Remuzzi, E. Marinoni, M. Marazzi, and R. Pietrabissa. Mechanobiology of engineered cartilage cultured under a quantified fluid-dynamic environment. Biomech. Model. Mechan., 1(1):69-82, 2002.

38. E. Sanchez-Palencia. Non-homogeneous Media and Vibration Theory, volume 127 of Lecture Notes in Physics. Springer-Verlag, 1980.

39. J.A. Sanz-Herrera, J.M. Garcia-Aznar, and M. Doblare. A mathematical model for bone tissue regeneration inside a specific type of scaffold. Biomech. Model. Mechan., 7(5):355-366, 2008.

40. B.G. Sengers. Modelling the development of tissue engineered cartilage. PhD thesis, Technische Universiteit Eindhoven, 2005. 
41. B.G. Sengers, M. Taylor, C.P. Please, and R.O.C. Oreffo. Computational modelling of cell spreading and tissue regeneration in porous scaffolds. Biomaterials, 28(10):1926-1940, 2007.

42. B.G. Sengers, C.C. van Donkelaar, C.W.J. Oomens, and F.P.T. Baaijens. Computational study of culture conditions and nutrient supply in cartilage tissue engineering. Biotechnol. Progr., 21(4):1252-1261, 2005.

43. B. Sharma and J.H. Elisseeff. Engineering structurally organized cartilage and bone tissues. Ann. Biomed. Eng., 32(1):148-159, 2004

44. R.J. Shipley. Multiscale Modelling of Fluid and Drug Transport in Vascular Tumours. PhD thesis, University of Oxford, 2009.

45. C.M. Smith, A.L. Stone, R.L. Parkhill, R.L. Stewart, M.W. Simpkins, A.M. Kachurin, W.L. Warren, and S.K. Williams. Three-dimensional bioassembly tool for generating viable tissue-engineered constructs. Tissue Eng., 10(9-10):1566-1576, 2004.

46. P. Sucosky, D.F. Osorio, J.B. Brown, and G.P. Neitzel. Fluid mechanics of a spinner-flask bioreactor. Biotechnol. Bioeng., 85(1):34-46, 2004.

47. M. Tomita, E.F. Sato, M. Nishikawa, Y. Yamano, and M. Inoue. Nitric oxide regulates mitochondrial respiration and functions of articular chondrocytes. Arthritis Rheum., 44(1):96-104, 2001.

48. P.A. Torzilli, E. Askari, and J.T. Jenkins. Water content and solute diffusion properties in articular cartilage. In V.C. Mow, A. Ratcliffe, and S.L-Y. Woo, editors, Biomechanics of Diarthrodial Joints, volume 1, chapter 13, pages 363-390. Springer, 1990.

49. X. Wang, Y. Yan, Y. Pan, Z. Xiong, H. Liu, J. Cheng, F. Liu, F. Lin, R. Wu, R. Zhang, and Q. Lu. Generation of three-dimensional hepatocyte/gelatin structures with rapid prototyping system. Tissue Eng., 12(1):83-90, 2006.

50. R. Whittaker, R. Booth, R. Dyson, C. Bailey, L. Parsons Chini, S. Naire, S. Payvandi, Z. Rong, H. Woollard, L. Cummings, S. Waters, L. Mawasse, J. Chaudhuri, M. Ellis, V. Michael, N. Kuiper, and S. Cartmell. Mathematical modelling of fibre-enhanced perfusion inside a tissue-engineering bioreactor. J. Theor. Biol., 256:533-546, 2009.

51. K.A. Williams, S. Saini, and T.M. Wick. Computational fluid dynamics modeling of steady-state momentum and mass transport in a bioreactor for cartilage tissue engineering. Biotechnol. Progr., 18(5):951-963, 2002.

52. T.B.F. Woodfield, J.M. Bezemer, J.S. Pieper, C.A. van Blitterswijk, and J. Riesle. Scaffolds for tissue engineering of cartilage. Crit. Rev. Eukar. Gene, 12(3):209-236, 2002.

53. T.B.F. Woodfield, J. Malda, J. de Wijn, F. Péters, J. Riesle, and C.A. van Blitterswijk. Design of porous scaffolds for cartilage tissue engineering using a three-dimensional fiber-deposition technique. Biomaterials, 25(18):4149-4161, 2004.

54. T.B.F. Woodfield, C.A. van Blitterswijk, J. de Wijn, T.J. Sims, A.P. Hollander, and J. Riesle. Polymer scaffolds fabricated with pore-size gradients as a model for studying the zonal organization within tissueengineered cartilage constructs. Tissue Eng., 11(9-10):1297-1311, 2005. 